\title{
Grid Convergence for Three Dimensional Benchmark Turbulent Flows
}

\author{
Boris Diskin* \\ National Institute of Aerospace (NIA), Hampton, VA 23666, USA
}

\author{
William K. Anderson†, Mohagna J. Pandya; Christopher L. Rumseyફ, James L. Thomas \\ NASA Langley Research Center, Hampton, VA 23681, USA \\ Yi Liu, Hiroaki Nishikawa** \\ National Institute of Aerospace (NIA), Hampton, VA 23666
}

\begin{abstract}
Grid convergence studies are performed to establish reference solutions for benchmark three dimensional turbulent flows in support of the ongoing turbulence model verification and validation effort at the Turbulence Modeling Resource website curated by NASA. The benchmark cases are a subsonic flow around a hemisphere cylinder and a transonic flow around the ONERA M6 wing with a sharp trailing edge. The study applies widely-used computational fluid dynamics codes developed and supported at the NASA Langley Research Center: FUN3D, USM3D, and CFL3D. Reference steady-state solutions are computed for the Reynolds-Averaged Navier-Stokes equations with the Spalart-Allmaras turbulence model on families of consistently-refined grids composed of different types of cells. Coarse-to-fine and code-to-code solution variation is described in detail.
\end{abstract}

\section{Introduction}

The Turbulence Modeling Resource (TMR) website ${ }^{1-3}$ supported at the NASA Langley Research Center has been widely used to validate and verify implementations of turbulence models. The validation cases offered at the website establish the ability of model equations to represent physics in a particular flow regime. Verification tests focus on solution-to-solution comparisons and establish correctness of specific implementations of turbulence models in computational fluid dynamics (CFD) codes. More recently, a new section, Cases and Grids for Turbulence Model Numerical Analysis, has been added to the TMR website. The test cases in this section are designed primarily for numerical analysis of solver technology used in turbulent flow simulations, e.g., grid and iterative convergence properties, effects of specific discretizations, grid-refinement strategies, etc. In support of the TMR objectives, special sessions on solver technology for turbulent flows were held at the AIAA Science and Technology Forum and Exposition (SciTech) conferences in $2015^{4-11}$ and 2016. ${ }^{12-20}$ Advanced solver technologies for the Reynolds-Averaged Navier-Stokes (RANS) equations with a one-equation linear eddyviscosity Spalart-Allmaras (SA) turbulence model ${ }^{21,22}$ were demonstrated in application to relatively simple benchmark flows in two dimensions (2D) and three dimensions (3D). Some of the solver-technology advances reported at these sessions, including adaptive and high-order solutions, have been published in a special AIAA Journal publication. ${ }^{23}$

Reference solutions are required to establish correctness and efficiency of new solver technologies. In the absence of exact solutions for RANS equations, the TMR approach to providing reference solutions is to conduct grid convergence studies and compare near zero-residual solutions obtained by several independent codes on

\footnotetext{
*NIA Research Fellow, Associate Fellow AIAA

$\dagger$ Senior Research Scientist, Computational Aerosciences Branch, Mail Stop 399, Associate Fellow AIAA

¥Research Aerospace Engineer, Configuration Aerodynamics Branch, Mail Stop 499, Senior Member AIAA

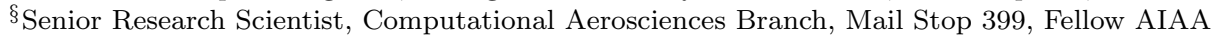

『Distinguished Research Associate, Computational Aerosciences Branch, Mail Stop 399, Fellow AIAA

$\|$ Senior Research Scientist, Senior Member AIAA

** NIA Associate Research Fellow, Associate Fellow AIAA
} 
consistently refined grids. The goal is to compute with sufficient degrees of freedom so that solutions computed by different codes on the finest grids are in good agreement; thereby establishing reference solutions. For the SciTech-2015 and SciTech-2016 benchmark flows, the reference solutions were computed by well-established CFD codes: FUN3D (NASA), USM3D (NASA), CFL3D (NASA), and TAU (DLR). Detailed descriptions of the reference solutions have been posted on the TMR website.

This paper extends the previous studies by providing reference solutions for turbulent flows around benchmark aerodynamic configurations that are the focus of a special session at SciTech-2018. A 3D hemispherecylinder configuration provides test cases for turbulent flows over a smooth body of revolution. The 3D ONERA M6 (OM6) configuration provides test cases for transonic turbulent flows over a swept wing. Specialized grid generation and grid coarsening codes have been developed for these studies to construct families of consistently refined grids that satisfy the current guidelines for grid convergence studies. ${ }^{24,25}$ Capabilities of these codes are described in an accompanying paper to be presented at the same SciTech-2018 special session. ${ }^{26}$ The RANS equations with the SA-neg variant ${ }^{22}$ of the SA turbulence model ${ }^{21}$ are solved for all flows considered in this paper. Three NASA CFD codes, FUN3D, USM3D, and CFL3D, are used in this study. The CFD codes use cell- and node-centered discretizations based on finite-volume and finite-element formulations. To exclude the influence of iterative errors, all solutions are converged to near-machine-zero residuals on all grids. The obtained reference solutions will be available on the TMR website.

Solutions are computed on structured and unstructured grids. Unstructured grids of four types, namely, fully prismatic grids, fully tetrahedral grids, mixed-element grids composed of prismatic and tetrahedral cells, and mixed-element grids composed of prismatic and hexahedral cells have been used in the current study. The grid generation and coarsening programs and representative input files are available at the TMR website. Unstructured grids of the same coarsening level have an identical distribution of grid nodes. Structured grids are hexahedral grids with some prismatic (degenerated hexahedral) cells around a polar singularity. Table 1 summarizes the grid family types.

Table 1: Grid families.

\begin{tabular}{cl} 
Grid family & Description \\
\hline 1 & unstructured, prismatic cells \\
2 & unstructured, tetrahedral cells \\
3 & unstructured, mixed-element prismatic and tetrahedral cells \\
4 & unstructured, mixed-element prismatic and hexahedral cells \\
5 & structured, hexahedral cells and prismatic cells at polar singularity \\
\hline
\end{tabular}

The material in the paper is presented in the following order. First, the discretization details of the codes used in the current study are outlined in Section II. Benchmark solutions for a subsonic turbulent flow around the hemisphere-cylinder configuration are described in Section III. Solutions corresponding to different discretization schemes are compared on several families of consistently-refined grids with degrees of freedom up to 420 million. Grid convergence of aerodynamic forces, surface and off-body solutions, and flow separation and reattachment locations are studied in detail and correlated with experimental measurements. Section IV reports on grid convergence studies for a transonic turbulent flow over an OM6 wing with a sharp trailing edge. The solutions are computed on consistently-refined grids with fine-grid degrees of freedom up to 360 million. Solutions computed by different codes on different grid families are compared with each other and with experimental measurements. Finally, conclusions are offered in Section V.

\section{CFD Codes}

This section describes the practical CFD codes used to establish reference solutions. The codes developed and supported at the NASA Langley Research Center are widely used by the U.S. government, industry, and academia and represent the state of the art in aerodynamic computations.

\section{II.A. FUN3D}

FUN3D is a node-centered, unstructured-grid RANS solver, which is widely used for high-fidelity analysis and adjoint-based design of complex turbulent flows. ${ }^{27-32}$ Two different discretizations available in FUN3D are employed: the baseline finite-volume discretization (FUNFV) and a recently implemented stabilized finite- 
element discretization (SFE) based on a Streamlined Upwind Petrov-Galerkin formulation. ${ }^{33}$

FUNFV discretizes the governing flow equations on mixed-element grids; the elements are tetrahedra, pyramids, prisms, and hexahedra. At median-dual control-volume faces, the inviscid fluxes are computed using an approximate Riemann solver. Roe's flux difference splitting (FDS) is used in the current study. For second-order accuracy, face values are obtained by a Monotonic Upwind Scheme for Conservation Laws (MUSCL) scheme, ${ }^{34}$ with unweighted least-squares gradients computed at the nodes. For this study, the MUSCL scheme coefficient is set to $\kappa=0.75$ for the meanflow equations on tetrahedral grids and $\kappa=0.5$ on grids of other families. The viscous fluxes use the full approximation of the viscous stresses. For tetrahedral grids, the viscous fluxes are discretized using the Green-Gauss (cell-based) gradients; this is equivalent to a Galerkin type approximation. For nontetrahedral grids, the edge-based gradients are combined with Green-Gauss gradients; this improves the $h$-ellipticity of the viscous operator. The velocity gradients used in evaluation of the SA turbulence model source term are computed using the Green-Gauss gradients evaluated on median-dual control volumes. The diffusion term in the SA turbulence model is handled in the same fashion as the meanflow viscous terms. In these studies, FUNFV uses a first-order approximation for the convection term in the SA turbulence-model equation.

SFE is a stabilized finite-element discretization recently added as a library to FUN3D. ${ }^{33}$ The implementation supports the same element types available with the FUNFV scheme, although only solutions on tetrahedral elements are shown in this paper. Although the implementation supports high-order spatial discretizations, only results with linear elements $\left(P_{1}\right)$ are included in this study. The SA turbulence model equation is discretized in the same way as the meanflow equations. As a result, the SFE discretization of the RANS equations is fully second order. The formulation includes a combination of a standard Galerkin discretization, stabilization terms to compensate for lack of streamwise dissipation, and penalty terms to enforce boundary conditions and to capture shocks.

For both FUN3D discretizations, adiabatic solid wall boundary conditions are approximated as strong specified-temperature boundary conditions. The wall temperature is taken as an approximate adiabatic-wall temperature normalized by the freestream temperature. The wall temperature does not change in iterations.

\section{II.B. USM3D}

USM3D is an unstructured-grid, finite-volume, cell-centered RANS solver ${ }^{35,36}$ that has been widely used within NASA, ${ }^{37-39}$ other U.S. government agencies, ${ }^{40}$ and industry ${ }^{41}$ as a workhorse for aerodynamic analysis of complex configurations. An enhanced mixed-element version of USM $3 \mathrm{D}^{42,43}$ is used for this study. A fully-implicit formulation is implemented, implying that the auxiliary solution variables at the grid nodes and boundary faces as well as the cell gradients are computed solely from the current solution variables defined at the cell centers. Solution values at the nodes are averaged from the solution values at surrounding cells. ${ }^{44-47}$ The second-order spatial discretization of inviscid fluxes is accomplished by reconstructing solutions at the cell faces; the reconstruction is based on solution gradients computed within cells. The reconstruction scheme corresponds to the MUSCL scheme with the MUSCL coefficient $\kappa=0$. For this study, the cell gradients are evaluated with the Green-Gauss integration. Inviscid fluxes are computed at each cell face with the upwind FDS scheme. Face gradients required for evaluation of viscous fluxes are computed from the Mitchell stencil. ${ }^{48,49}$ The convection term of the SA turbulence model equation is approximated with first-order accuracy. The velocity gradients contributing to the source term of the SA model are computed at cell centers as the face-area average of face gradients. The adiabatic solid wall boundary conditions are implemented in a weak form.

\section{II.C. CFL3D}

CFL3D is a structured-grid, multiblock, cell-centered, finite-volume code widely applied for analysis of complex flows. It has been used for computing benchmark solutions in many recent workshops involving complex turbulent flows ${ }^{12,50-53}$ and at the TMR website. It uses second-order, upwind-biased spatial differencing scheme (a MUSCL scheme with the MUSCL coefficient $\kappa=1 / 3$ ) that provides third-order accuracy in one dimension for the convection and pressure terms. A second-order differencing is used for the viscous terms. Therefore, the meanflow discretization is globally second-order accurate. Roe's FDS method is used to obtain inviscid fluxes at the cell faces. The option to model the full Navier-Stokes meanflow equations is exercised for all cases. In this study, CFL3D uses a first-order approximation for the convection term in the SA model. The turbulence-model diffusion term uses a thin-layer approximation. The adiabatic solid wall boundary conditions are implemented in a weak form. 


\section{Subsonic Flow around a Hemisphere Cylinder}

Reference solutions for a flow around a 3D hemisphere-cylinder configuration are presented in this section. The study corresponds to the case described under the "3D Hemisphere Cylinder (new)" subsection of the "Cases and Grids for Turbulence Model Numerical Analysis" section at the TMR website. The reference solutions computed by FUNFV, SFE, USM3D, and CFL3D are used to verify RANS solvers for a turbulent flow over a smooth $3 \mathrm{D}$ body of revolution. A comparison with an experimental study ${ }^{54}$ provides a basis for the SA-neg turbulence model validation.

\section{III.A. Geometry, Flow Parameters, and Boundary Conditions}

The geometry is taken from the experimental study reported by Tsieh. ${ }^{54}$ In the experiment, the radius of the hemisphere was 0.5 in., the body length was 10 in., and the unit Reynolds number per foot was $4.2 \times 10^{6}$. Thus, in the computational domain with the unit length taken as $1 \mathrm{in}$., the hemisphere radius is 0.5 , the cylinder length is 9.5 , and the Reynolds number is $R e=3.5 \times 10^{5}$ per unit length. The reference solutions posted at the TMR website have been computed at the following flow conditions: the reference Mach number $M_{r e f}=0.6$, angles of attack of $0^{\circ}, 5^{\circ}, 10^{\circ}, 15^{\circ}$, and $19^{\circ}$, and the reference temperature $T_{r e f}=540^{\circ} \mathrm{R}$. This paper presents only solutions corresponding to the $19^{\circ}$ angle of attack.

The origin of the coordinate system is located at the apex of the hemisphere. The positive $x$ direction is the streamwise direction colinear with the axis of the hemisphere and cylinder. Figure 1 sketches the layouts of boundary conditions and shows the global view of a computational grid with half-plane symmetry. The downstream computational boundary is located at the back of the cylinder, $x=10$. The outflow conditions specified at the downstream boundary are constant pressure conditions corresponding to $P / P_{\text {ref }}=1$. The farfield boundary is a hemisphere with radius of 100 units centered at $x=10, y=0, z=0$.

\section{III.B. Grids}

Table 2: Statistics of four finest grids for hemisphere cylinder grid families.

\begin{tabular}{rrrrrrr}
\hline \multirow{2}{*}{ Grid } & \multicolumn{1}{c}{ Family 2 (tet) } & \multicolumn{1}{c}{ Family 4 (prism/hex) } & \multicolumn{2}{c}{ Family 5 (struct) } \\
\cline { 2 - 7 } & \multicolumn{1}{c}{ Cells } & \multicolumn{1}{c}{ Nodes } & \multicolumn{1}{c}{ Cells } & \multicolumn{1}{c}{ Nodes } & \multicolumn{1}{c}{ Cells } & \multicolumn{1}{c}{ Nodes } \\
\hline L1 & $424,673,280$ & $71,368,353$ & $78,643,200$ & $71,368,353$ & $78,643,200$ & $79,115,841$ \\
L2 & $53,084,169$ & $8,995,153$ & $9,830,400$ & $8,995,153$ & $9,830,400$ & $9,948,961$ \\
L3 & $6,635,520$ & $1,143,081$ & $1,228,800$ & $1,143,081$ & $1,228,800$ & $1,258,641$ \\
L4 & 829,440 & 147,637 & 153,600 & 147,637 & 153,600 & 161,161 \\
\hline
\end{tabular}

Five grid families are generated for this study. Figures 2, 3, and 4 illustrate grids of families 2 (tet), 4 (prism/hex), and 5 (struct), respectively, with a symmetry plane at $y=0$. Table 1 provides a summary of grid elements. Unstructured grids of families 1 to 4 have triangular faces on the hemisphere surface and no polar singularity. Each family has four levels of nested grids; L1 is the finest grid level, L2 is the second finest grid level, etc. Statistics of grids from families 2 (tet), 4 (prism/hex), and 5 (struct) are shown in Table 2. Family 5 (struct) includes structured grids with quadrilateral faces on the hemisphere surface. These grids have a polar singularity at the axis attached to the apex of the hemisphere, i.e., along this polar axis, hexahedral cells degenerate into prismatic cells. Unstructured grids corresponding to the same level have the same distribution of grid nodes. In comparison with unstructured grids at the same level, family 5 (struct) grids have the same number of surface elements on the hemisphere surface, the same distribution of nodes on the cylinder surface, and more nodes on the hemisphere surface. All the L1 grids have the near-surface normal spacing approximately corresponding to $y^{+}=0.5$. FUNFV solutions are computed on grids of families 2 (tet) and 4 (prism/hex); SFE solutions are computed on family 2 (tet) grids, USM3D solutions are computed on grids of families 2 (tet) and 5 (struct), and CFL3D solutions are computed on family 5 (struct) grids.

\section{III.C. Results}

\section{III.C.1. Solution visualization}

For solution visualization, Fig. 5 presents the FUN3D solution computed on the prism/hex L1 grid of family 4. The pressure contours and streamlines are shown in four planes corresponding to $y=0, z=0, x=5.0$, and 
$x=6.0$. The pressure is nondimensionalized by $\rho_{r e f} a_{r e f}^{2}$, where $\rho_{r e f}$ and $a_{r e f}$ are the dimensional freestream density and speed of sound, respectively. In the symmetry plane corresponding to $y=0$, the cross-stream separation is characterized by downward flow velocity. The separation occurs behind the hemisphere-cylinder junction and continues for the entire cylinder length. A minimum pressure is observed on the leeside, upstream of the hemisphere-cylinder junction. A regular flow pattern with no separation is seen in the plane corresponding to $z=0$. A large primal vortex and a smaller secondary vortex are shown in the cross flow planes corresponding to $x=5.0$ and $x=6.0$. The separation locations of these primal and secondary vortices are similar to those documented in the experiment. An off-body vortex is seen in the shear layer of the primal vortex, outboard of the secondary one.

\section{III.C.2. Forces and pitching moment}

Table 3: Hemisphere Cylinder: Variation of aerodynamic coefficients computed on L1 grids.

\begin{tabular}{ccccc}
\hline $\begin{array}{c}\text { Aerodynamic } \\
\text { Coefficient }\end{array}$ & $\begin{array}{c}\text { Variation } \\
\text { (range) }\end{array}$ & $\begin{array}{c}\text { Variation } \\
(\%)\end{array}$ & $\begin{array}{c}\text { Core Variation } \\
\text { (range })\end{array}$ & $\begin{array}{c}\text { Core Variation } \\
(\%)\end{array}$ \\
\hline lift & $0.08535-0.08692$ & $1.82 \%$ & $0.08607-0.08628$ & $0.24 \%$ \\
total drag & $0.03444-0.03490$ & $1.33 \%$ & $0.03453-0.03469$ & $0.46 \%$ \\
pressure drag & $0.02177-0.02234$ & $2.58 \%$ & $0.02198-0.02213$ & $0.68 \%$ \\
viscous drag & $0.01254-0.01268$ & $1.11 \%$ & $0.01254-0.01256$ & $0.16 \%$ \\
pitching moment & $(-0.02650)-(-0.02536)$ & $4.40 \%$ & $(-0.02588)-(-0.02569)$ & $0.74 \%$ \\
\hline
\end{tabular}

Grid convergence plots of the lift, total drag (including pressure and viscous components), and pitching moment coefficients and the maximum eddy viscosity are shown in Fig. 6. The plotted eddy viscosity is nondimensionalized by $\mu_{r e f}$, which is the laminar viscosity of the freestream flow conditions. The value of the characteristic grid spacing, $h$, is computed as $h=N^{-1 / 3}$, where $N$ is the number of degrees of freedom (cells for USM3D and CFL3D, nodes for FUNFV and SFE). The aerodynamic coefficients computed with different codes on different grid families are generally converging to the same limit with grid refinement. Convergence of the maximum eddy viscosity is less clear, mainly because of the disagreement between limit projections from FUNFV (prism/hex) solutions and other solutions, even though the SFE and FUNFV (prism/hex) solutions agree well on the finest L1 grids.

Overall code-to-code aerodynamic-coefficient variation from the L4 grids to the $\mathrm{L} 1$ grids is up to $20 \%$. In this estimate and in the rest of the paper, relative variation is computed with respect to the middle of the variation range. Extrapolation to the infinite-grid limit is problematic because no reliable order of convergence can be established. No solution appears to converge uniformly in all quantities. Three solutions, USM3D (struct), FUNFV(prism/hex) and SFE, converge monotonically. Considering lift, USM3D (struct) solutions show less than first-order convergence, i.e., the lift approaches the limit from above with a concave shape. The FUNFV (prism/hex) and SFE lift curves approach the limit from above with convex shapes, indicating a convergence order that is higher than first order. The FUNFV (tet) lift appears to converge with first order on the three finer grids. The USM3D (tet) lift converges from above and changes the curve shape from concave to convex. Considering pressure drag convergence, the FUNFV (prism/hex) and SFE convergence curves approach the limit with convex shapes from above, but intersect. Lacking an exact solution, we use a quantitative characterization of observed solution variation to evaluate accuracy. Variation of the aerodynamic coefficients computed on the L1 grids is described in Table 3. The largest relative difference among all solutions is observed for the pitching moment and does not exceed $4.4 \%$.

Accuracy of aerodynamic coefficients improves proportionally to degrees of freedom used in CFD computations. This property is the foundation of all grid refinement studies. It also justifies the expectation of accuracy benefits from tetrahedral-grid cell-centered formulations that provide more degrees of freedom on grids of the same level. The USM3D solutions use about six times more degrees of freedom on grids of family 2 (tet) than other solutions on grids of the same level. Because grid convergence shown in Fig. 6 is not regular, quantitative assessments of accuracy improvements due to additional degrees of freedom are difficult and imprecise. Qualitatively, the aerodynamic coefficients computed by USM3D(tet) on the L2 grid are within the variation range of the L1 solutions.

Looking at the grid convergence on the three finer grids, the maximum and minimum values of integrated aerodynamic quantities have been generally exhibited by the CFL3D solutions and the FUNFV (tet) solutions. 
(The only exception is that, for the viscous drag coefficient, the minimum is exhibited by the SFE solutions.) The L1 solutions of remaining codes are highly clustered. We have defined a core group as consisting of these solutions, namely, the USM3D (struct), USM3D (tet), FUNFV(prism/hex), and SFE solutions. Relative variation among the core-group L1 solutions is also shown in Table 3. Variation among the core-group solutions is significantly smaller than variation among all solutions and does not exceed $0.74 \%$.

There are two possible reasons for differences among solutions obtained in the limit of grid refinement: (1) CFL3D uses a thin-layer approximation for the diffusion term in the SA model equation, while all other codes use the full Navier-Stokes approximation; and (2) FUNFV and SFE use an adiabatic-wall condition that is different from the one used by USM3D and CFL3D. The deviations of the CFL3D solutions from the core-group solutions may be attributed to the thin-layer approximation. The deviations in the FUNFV (tet) solutions observed on the current grids are harder to explain. In the limit of grid refinement, all FUNFV and SFE solutions are expected to converge to the same "infinite-grid" solution. On the current grids, nonphysical oscillatory solution modes resembling checker-board instabilities were observed in the FUNFV (tet) solutions with the default MUSCL scheme coefficient, $\kappa=0.0$. The FUNFV solutions computed on grids of other families are smooth. Note that the default value of the MUSCL scheme coefficient on nontetrahedral grids is $\kappa=0.5$. In this study, an increased coefficient of $\kappa=0.75$ is used for FUNFV (tet) solutions. Solutions with $\kappa=0.75$ do not exhibit nonphysical oscillations, but appear to be somewhat less accurate. Although not shown, FUNFV solutions with $\kappa=0.0$ were computed on tetrahedral grids by using the approximate mapping discretization method for inviscid fluxes. ${ }^{55,56}$ Approximate-mapping solutions do not exhibit nonphysical oscillations and provide aerodynamic coefficients well within the core-group variation range.

\section{III.C.3. Fine grid surface pressure, skin friction, and off-body variation}

In this section, surface pressure and skin friction are shown for four sets of solutions: USM3D (tet), FUNFV(prism/hex), SFE, and CFL3D solutions. Hereafter, only these four hemisphere-cylinder solutions of the available set are shown mainly for conciseness and presentation clarity. First, global views of solution variation on the L1 grids are shown. Figure 7 shows the surface pressure and the $x$-component of skin friction at the symmetry plane corresponding to $y=0$. The pressure maximum indicating the leading edge stagnation is located near $x=0.03$ on the windward side of the hemisphere. The pressure minima are observed on the leeside near $x=0.3$ and on the windward side near $x=0.45$. A zone of low pressure is also observed on the leeside at $x>4$. Near the outflow boundary, the leeside pressure increases and the windward pressure decreases, creating a small negative-lift zone. The L1-grid surface pressure distributions computed with different codes are almost indistinguishable. The fine-grid code-to-code differences in the surface pressure at the local extrema located on the hemisphere are within $0.5 \%$. Although comparisons with experimental data are not the focus of this paper, the computed surface pressure agrees qualitatively with the experimental measurements. ${ }^{54}$ Figure $7(\mathrm{~b})$ shows the $x$-component of the skin friction vector. Note that the $y$ - and $z$-components of the skin friction are zero at the symmetry plane. The skin-friction profiles computed with different codes are similar in most places. The largest discrepancy of about $15 \%$ is observed on the leeside, near $x=6.5$. Other places of noticeable but more local discrepancies are downstream from the hemisphere-cylinder junction at $x=0.5$ and near the outflow boundary.

Figure 8 shows the L1 surface pressure and $x$-direction and tangential components of skin friction at the $z=0$ plane. The tangential direction is defined on the hemisphere-cylinder surface as a unit vector tangential to the surface, normal to the $x$-direction, and pointing up (having a positive $z$-component). The tangential direction is uniquely defined almost everywhere on the surface, except at the symmetry plane. The tangential component of skin friction is the projection of the skin-friction vector onto the tangential direction. The surface pressure and skin-friction components computed by different codes are almost indistinguishable in the global view. Some differences (about 10\%) can be seen only in the tangential skin-friction component (Fig. 8(c)) near the junction between the hemisphere and cylinder located at $x=0.5$. The computed pressure profiles agree well with the experiment.

L1-grid variation of the surface pressure and $x$-component and tangential component of skin friction in the plane corresponding to $x=5$ are shown in Fig. 9. Variation is shown versus the circumferential angle computed

as $\phi=\cos ^{-1}\left(z / \sqrt{y^{2}+z^{2}}\right) ; \phi=0^{\circ}$ corresponds to the leeside $(y=0, z>0), \phi=90^{\circ}$ corresponds to the horizontal plane $(y>0, z=0)$, and $\phi=180^{\circ}$ corresponds to the windward side $(y=0, z<0)$. The surface pressure and skin-friction profiles computed with different codes on L1 grids of different families are almost overplotted, except for the differences (less than 10\%) observed for the $x$-component of skin friction near the leeside. This region is close to the location that shows the largest discrepancies on Fig. 7(b). The change of sign 
in the tangential component of skin friction indicates crossflow vortex separation and reattachment. The flow in the $x=5$ plane features two vortices: the primal vortex rotating in the counterclockwise direction separates at $\phi \approx 65^{\circ}$; the secondary vortex rotating clockwise separates at $\phi \approx 30^{\circ}$ and reattaches at $\phi \approx 42^{\circ}$.

Figure 10 shows a global view of off-body profiles of the pressure and velocity components computed with different codes on the L1 grids. The pressure is nondimensionalized by $\rho_{r e f} a_{r e f}^{2}$, and the velocity components are nondimensionalized by $a_{r e f}$. The solutions are plotted along a vertical line attached to the upper surface of the cylinder at $x=5$ and $y=0.21$. The view is chosen to show solution variation across the core of the primal crossflow vortex located in this area. All off-body profiles are overplotted in the global view.

\section{III.C.4. Grid refinement of surface pressure, skin friction, and off-body variation}

Grid refinement and zooming have been applied to study solution variation near nontrivial flow features and near regions of the largest solution differences observed in global views. Although not shown, global views of the surface pressure profiles in the three planes $(y=0, z=0$, and $x=5)$ computed on four grids in corresponding families are hardly distinguishable. Figure 11 illustrates local grid convergence of the leeside surface pressure near the location of the minimum pressure. Even in the zoomed view, only the coarsest L4 grid solutions are clearly distinguishable. Figure 12 illustrates grid convergence of the minimum pressure on the leeside and on the windward side. All codes appear to approach the same limit. Variation between L1 solutions is less than $0.07 \%$. The minimum pressure coefficients computed with USM3D, FUNFV, and CFL3D decrease monotonically with grid refinement and show similar coarse-to-fine grid variation of about $7 \%$. The SFE minimum pressure coefficients show remarkably small coarse-to-fine grid variation of less than $0.5 \%$, but converge nonmonotonically with grid refinement.

Grid convergence plots zoomed to a vicinity of the outflow boundary shown in Fig. 13 indicate that the leeside surface pressure coefficients computed on L1 and L2 grids are close everywhere except for the last point located at the outflow boundary. Note that the plotted CFL3D pressure coefficients are computed at the face centers; all other solutions provide surface pressure coefficients at nodes. Figure 14 shows the details of grid convergence of the leeside surface pressure coefficient at two locations corresponding to $x=9.0$ and $x=9.5$. SFE has the least coarse-to-fine variation. No order property is evident in any of the solutions. At $x=9.0$, the largest discrepancy between L1 solutions is approximately 0.003 , which is about $30 \%$; at $x=9.5$, the largest L1 discrepancy is close to 0.007 , which is also about $30 \%$. This relative difference is the largest that we observed, which is in part attributed to small solution values and a proximity to the outflow that is expected to have a singularity. The CFL3D fine-grid pressure coefficient is lower than the coefficients computed from other solutions, especially at $x=9.5$. This deviation may be caused by a combination of factors, such as the thin-layer diffusion approximation in the SA model and different (slightly off the symmetry plane) locations of computed pressure coefficients.

Figure 15 shows grid convergence of the leeside skin-friction $x$-component near the location of the largest discrepancies between L1-grid solutions (cf. Fig. 7(b)). There are significant (up to 20\%) differences between L1 solutions computed with different codes and L4-to-L1 solutions computed with the same code. The differences are both qualitative and quantitative. The L4 and L3 USM3D, FUNFV, and CFL3D solutions do not predict a local skin-friction minimum in the area $4 \leq x \leq 8$. The L2 and L1 solutions computed with the same codes predict a local minimum. The SFE solutions on all grids predict a local minimum of skin friction. Generally, all finer-grid solutions approach a local minimum of $C_{f, x} \approx 0.0053$ near $x \approx 6.5$. The SFE solutions converge to this limit from below; the USM3D, FUNFV, and CFL3D solutions converge to this limit from above. The current L1 grids do not provide sufficient resolution to establish this limit behavior with certainty. Note that the discrepancies between skin-friction levels are well localized within the zoom interval; the skin-friction levels agree well at the ends of the zoom interval, $x \approx 4$ and $x \approx 8$. The abrupt change in the skin-friction profile near $x \approx 6.5$ is not well understood. The SA model primary variable is growing rapidly in this region. The secondorder approximation to the convection term of the SA model equation that is employed by SFE is believed to be a significant factor improving solution accuracy in this region and allowing detection of the local skin-friction minimum on coarse grids. Figure 16 provides another zoomed view of variation of the $x$-direction skin-friction component in approximately the same region. Grid convergence is shown in the $x=5$ plane near $\phi=0$ corresponding to the leeside. Similar to Fig. 15, there are significant differences between coarse- and fine-grid skin-friction profiles near the leeside. The SFE solutions exhibit much smaller fine-to-coarse grid variation, but the L1 SFE solution is significantly different from other L1 solutions. The L1 SFE solution indicates a local skin-friction minimum near $\phi \approx 6^{\circ}$, all other profiles do not show this minimum. Away from the leeside, all codes appear to be converging to the same limit.

Although not shown, grid convergence studies in another region, $0 \leq x \leq 1.0$, where skin friction differences 
are visible in a global view (Fig. 7(b)), reveal a more regular behavior. The skin friction profiles have been considered in the planes corresponding to $y=0$ and $z=0$. The L2 and L1 profiles are close for all codes and solutions on L3, L2, and L1 grids become close in regions $x<0.2$ and $x>0.8$. Skin friction profiles from all solutions appear to be converging with grid refinement to the same profile, with a minimum skin friction located at $x=0.5$.

Figures 17 and 18 show grid convergence of the pressure and the $x$-component of the skin friction evaluated within the $x=5$ plane near the local pressure minimum located at $\phi \approx 97.5^{\circ}$. Both pressure and skin friction appear to be converging to the same limit solution. Figure 19 shows pointwise convergence of the pressure coefficient and the $x$-component of skin friction at $\phi=97.5^{\circ}$. The L1 pressure range is less than $1 \%$; the corresponding L1 range of the $x$-component of skin friction is less than $0.5 \%$. In spite of such a close agreement between the L1 solutions, the asymptotic convergence cannot be established for all solutions. Although indiscernible from the figure, the pressure convergence plots observed for SFE and FUNFV solutions are not monotonic, and convergence plots corresponding to different codes intersect. Figure 20 illustrates grid convergence of the separation/reattachment angles for the primal and secondary vortices within the $x=5.0$ plane. An excellent grid convergence is observed. The separation/reattachment angles evaluated from solutions of different codes on L1 grids differ by 1 degree at most. The report ${ }^{54}$ provides a plot of separation angles observed in the experiment versus the $\mathrm{x}$ location. The experimental separation angles are also shown in Fig. 20 for reference.

Figures 21-24 show a view of grid refinement of off-body solution components near the upper surface at $x=5.0$. All solutions converge to the same profiles in the limit of grid refinement. Large solution variation seen near the leeside in Figs. 15 and 16 are not observed, attributed to the fact that this location that is farther from the symmetry plane.

\section{Transonic Flow around an OM6 Wing}

The ONERA M6 experiment ${ }^{57}$ has been widely used for validation of CFD solvers. A relatively simple, well-documented geometry and a rich experimental database for a large variety of flow conditions provide a unique combination for practical and inexpensive benchmark studies. The TMR website complements the ONERA efforts by providing grid generation capabilities and reference solutions for such benchmark studies. Reference solutions for transonic flows around the OM6 wing are presented in this section. Authors believe that the solutions computed on grids with more than 360 million degrees of freedom represent the largest OM6 computations conducted to date. The grid generation, coarsening, partition, and multigrid capabilities for the OM6 model are described in an accompanying paper. ${ }^{26}$ The OM6 wing geometry used in this study has been slightly redefined for numerical analysis of turbulence model simulations.

\section{IV.A. Geometry, Flow Parameters and Boundary Conditions}

Recently, a group at ONERA has considered the M6 model and its past experiments in greater detail. ${ }^{5,17}$ As part of this effort, the group has created a new CAD geometry for the wing. In this geometry, the trailing edge of the wing has been made sharp for the purpose of this particular CFD exercise. ${ }^{17}$ The original OM6 wing has a moderately thick trailing edge. Additional details are provided at the TMR website.

The reference solutions for the OM6 wing are computed at a freestream Mach number 0.84, Reynolds number $14.6 \times 10^{6}$ based on the unit root chord, and the angle of attack of $3.06^{\circ}$. The experimental data shown in this section corresponds to the case $2308,5,17,57$ of the ONERA S2MA wind tunnel test. The case 2308 is a relatively easy case exhibiting an attached transonic flow with two merging shocks. The farfield boundary in the shape of a hemisphere is located at 100 unit chords. The symmetry condition is assigned at the plane containing the root airfoil. Note that the experiment used a splitter plate near the wing root, which is not modelled by CFD codes. This discrepancy is believed to be the cause of disagreement between CFD solutions and experiment measurements at inboard sections.

\section{IV.B. Grids}

The OM6 grids used in this study are topologically equivalent to the full-geometry (no $y=0$ symmetry plane) hemisphere-cylinder grids described in Section III.B. The cylinder surface is mapped on the wing surface with the specified wing section, and the hemisphere surface is mapped on the rounded wing tip. Five nested grid families (Table 1) have been generated for the OM6 geometry by using input files available at the TMR website. Statistics of L4-L1 grids from families 1 (prism), 2 (tet), 4 (prism/hex) and 5 (struct) are shown in Table 4. 
Table 4: Statistics of grids for OM6 wing grid families.

\begin{tabular}{|c|c|c|c|c|c|c|c|c|}
\hline \multirow{2}{*}{ Grid } & \multicolumn{2}{|c|}{ Family 1 (prism) } & \multicolumn{2}{|c|}{ Family 2 (tet) } & \multicolumn{2}{|c|}{ Family 4 (prism/hex) } & \multicolumn{2}{|c|}{ Family 5 (struct) } \\
\hline & Cells & Nodes & Cells & Nodes & Cells & Nodes & Cells & Nodes \\
\hline L1 & $121,110,528$ & $60,777,345$ & $363,331,584$ & $60,777,345$ & $69,206,016$ & $60,777,345$ & $69,206,016$ & $69,305,025$ \\
\hline $\mathrm{L} 2$ & $15,138,816$ & $7,625,153$ & $45,416,448$ & $7,625,153$ & $8,650,752$ & $7,625,153$ & $8,650,752$ & $8,675,681$ \\
\hline L3 & $1,892,352$ & 960,225 & $5,677,056$ & 960,225 & $1,081,344$ & 960,225 & $1,081,344$ & $1,087,665$ \\
\hline $\mathrm{L} 4$ & 236,544 & 121,841 & 709,632 & 121,841 & 135,168 & 121,841 & 135,168 & 136,793 \\
\hline
\end{tabular}

On the L1 grids, there are 384 nodes along each wing section, 192 nodes/quadrilateral elements in the spanwise direction excluding the rounded tip, 24576 surface elements over the tip, and 704 nodes/cells in the direction normal to the wing surface. Figure 25 illustrates OM6 grids of families 4 (prism/hex) and 5 (struct) with a close view on the wing tip surface. The farfield boundary grids are not shown because they look similar to the full-geometry extension of those for the hemisphere-cylinder configuration (Figs. 3 and 4). The surface grids have a moderate stretching toward the leading and trailing edges resulting in a relatively coarse grid spacing in the midchord region. All the L1 grids have the first node off the surface located at an average of approximately $y^{+}=0.5$.

\section{IV.C. Results}

Figure 26 presents the contours of the surface pressure computed by USM3D on the prism/hex L1 grid of family 4 . The pressure is nondimensionalized by $\rho_{r e f} a_{r e f}^{2}$. A lambda shock is clearly visible on the surface with the shock intersection located at about $80 \%$ of the wingspan.

Grid convergence of aerodynamic coefficients is described next. USM3D solutions have been computed on grids of families 2 (tet) and 4 (prism/hex); FUNFV solutions have been computed on grids of families 1 (prism) and 4 (prism/hex); and CFL3D solutions have been computed on structured grids of family 5 (struct). All computations have been conducted with no flux limiters. Figures $27(\mathrm{a})-27(\mathrm{~d})$ show convergence of the lift, total drag, pressure drag, viscous drag, and pitching moment coefficients and the maximum eddy viscosity. No solution converges monotonically for all plotted quantities; thus, no order property can be deduced from the observed convergence. Nevertheless, all solutions approach the same aerodynamic coefficient values in the limit of grid refinement. The slopes of the lift and pitching moment convergence curves shown in Figs. 27(a) and $27(\mathrm{e})$ are highly irregular for solutions on grid families 4 (prism/hex) and 5 (struct). For example, the pitching moment coefficient computed from the family 4 USM3D (prism/hex) solutions decreases initially with grid refinement from L4 grid to L3 grid, increases on L2 grid, and decreases again on L1 grid. Lift and pitching moment convergence observed for FUNFV (prism) and USM3D (tet) solutions is more regular. The differences among lift and pitching-moment coefficients computed by all codes on all grids do not exceed $6 \%$.

Drag coefficients shown in Figs. 27(b)-27(d) appear to be converging with more regular slopes, but do not provide convergence patterns suitable for the infinite-grid extrapolation. The total and pressure drag coefficients computed from CFL3D and FUNFV solutions change the direction of convergence on the L1 grids. The viscous drag coefficient computed from the USM3D (tet) solution changes the direction of convergence on the L1 grid. Only USM3D (prism/hex) solutions converge monotonically for the three drag coefficients. Relative variation of drag coefficients computed on different grids is more significant than variation of the lift and pitching moment coefficients; pressure and viscous drag coarse-to-fine variation is approximately $30 \%$ and $16 \%$, respectively. Relative coarse-to-fine variation of the maximum eddy viscosity shown in Fig. 27(f) is too large to establish a grid-converged limit with any reasonable certainty.

To establish solution accuracy, Table 5 shows code-to-code variation of the forces, pitching moment, and maximum eddy viscosity on the L1 grid. Among all integral aerodynamic coefficients, the maximum relative difference of $0.94 \%$ is observed for the pressure drag. Maximum eddy viscosity variation exceeds $10 \%$, indicating considerably higher uncertainty than in integrated quantities.

The accuracy impact of increased degrees of freedom offered by cell-centered formulations on tetrahedral grids is qualitatively similar to the one observed for the hemisphere-cylinder computations: aerodynamic coefficients computed by USM3D (tet) on the L2 grid are close to aerodynamic coefficients computed from L1 solutions with similar degrees of freedom. For quantities that converge regularly in grid refinement, e.g., lift (Fig. 27(a)) and pitching moment (Fig. 27(e)), the USM3D (tet) solution appears to provide significant accuracy benefits on same-level grids.

Variation of surface pressure coefficients computed on the L1 grids at the measurement sections used in the 
Table 5: Variations of aerodynamic coefficients.

\begin{tabular}{ccc} 
Aerodynamic Coefficient & Variation (values) & Variation (\%) \\
\hline lift & $0.2692-0.2706$ & $0.52 \%$ \\
total drag & $0.01691-0.01705$ & $0.82 \%$ \\
pressure drag & $0.01163-0.01174$ & $0.94 \%$ \\
viscous drag & $0.005279-0.005309$ & $0.57 \%$ \\
pitching moment & $(-0.1912)-(-0.1898)$ & $0.73 \%$ \\
max eddy viscosity & $1386-1536$ & $10.27 \%$ \\
\hline
\end{tabular}

Table 6: Locations of surface pressure data, wingspan $b=1.47601797621980$.

\begin{tabular}{ccccc} 
Section & $\eta=y / b$ & $y$ & $x_{\min }$ & $x_{\max }$ \\
\hline 1 & 0.20 & 0.295203595243960 & 0.170429006219 & 1.0829321146 \\
2 & 0.44 & 0.649447909536712 & 0.374943822622 & 1.18245089054 \\
3 & 0.65 & 0.959411684542870 & 0.553894281387 & 1.26952958107 \\
4 & 0.80 & 1.18081438097584 & 0.681716024876 & 1.33172881603 \\
5 & 0.90 & 1.32841617859782 & 0.766930520535 & 1.37319493294 \\
6 & 0.96 & 1.41697725717101 & 0.818059265614 & 1.39807462692 \\
7 & 0.99 & 1.46125779645760 & 0.843623578548 & 1.41051435471 \\
\hline
\end{tabular}

experiment $^{5,17,57}$ is shown in Fig. 28. Only three computations, FUNFV (prism/hex), USM3D (prism/hex), and CFL3D (struct), are used in this section for conciseness. The three codes extract surface pressure at the same spanwise locations specified in Table 6. The wingspan is taken as $b=1.47601797621980$ and the relative axial position was computed as $x / c=\left(x-x_{\min }\right) /\left(x_{\max }-x_{\min }\right)$. In the global view, the L1 pressure profiles from different codes are in close agreement. Small oscillations in FUNFV solutions are observed near the shocks at sections 1-4. All solutions place shocks at the same locations and identify the same pressure minima on the lower and upper wing surfaces. The pressure profiles at leading and trailing edges are indistinguishable. As compared to other studies, ${ }^{5,17}$ an improved agreement with the experiment is observed at section 4 . This improvement is observed to be due to the increased grid resolution provided by the L1 grids.

Figures 29-31 show a global view of leeside pressure grid refinement at sections 1, 4, and 7, respectively. The pressure plots show significant variation with grid refinement. The midchord grid spacing on L4 and L3 grids is too coarse to represent details of the pressure profiles; the corresponding coarse-grid solutions miss most of the shock structure and are significantly different from the solutions obtained on the fine grids. All solutions computed on L2 and L1 grids represent the shock details and agree to each other remarkably well. The grid convergence patterns of USM3D and CFL3D solutions are quite similar, as expected, because both codes use cell-centered formulations. FUNFV uses a node-centered formulation and exhibits a different convergence pattern.

Figures 32-35 focus on grid convergence in the vicinity of remarkable geometry and flow features, such as trailing edge, minimum of pressure, and shocks. Figure 32 shows grid convergence of upper surface pressure at the most inboard section 1 of the OM6 wing, near the trailing edge in the interval corresponding to $0.98<x / c<1.0$. The L4 solutions are significantly different from solutions on the three finer grids. The solutions from the same codes computed on the L3-L1 grids are close; L3-to-L1 variation on this interval does not exceed $4.2 \%$ except at the trailing edge itself. The code-to-code discrepancy diminishes with grid refinement. At the trailing edge, the USM3D and CFL3D solutions converge from above; the FUNFV solution converges from below. All solutions away from trailing edge converge from above. Variation between L1 solutions at the point immediately preceding the trailing edge, $x / c \approx 0.999974$, is about $1.8 \%$. L1 solution variation is rapidly decreasing with the distance from the trailing edge. At $x / c \approx 0.98$, variation is $0.15 \%$.

At the trailing edge point, the code-to-code discrepancy diminishes with grid refinement, but is still significant; the difference between trailing-edge pressure in L1 solutions is about $11 \%$. While the L3-L1 solutions are close, no conclusion can be reached about convergence order. The difference between the FUNFV L1 and L2 solutions is larger than the difference between the L2 and L3 solutions; the difference between the USM3D L1 and L2 solutions is smaller than the difference between the L2 and L3 solutions; and the difference between the CFL3D L1 and L2 solutions is about the same as the difference between the L2 and L3 solutions. 
Figure 33 shows grid convergence of surface pressure at section 1 near the minimum-pressure location in the interval corresponding to $0.015<x / c<0.065$. The solutions rapidly converge with grid refinement, resulting in large coarse-to-fine grid variation. Coarse-to-fine grid variation upstream of the minimum-pressure location is much smaller than in the immediate vicinity and downstream of this location. The L4 solutions differ from the L1 solutions by more than $30 \%$. The L3 solutions differ from the L1 solutions by about 10\%, although the FUNFV L3 solution identifies the minimum of pressure accurately. The L1 solutions agree well with each other. All codes identify the minimum of pressure at the same location, $x / c \approx 0.39$. The code-to-code discrepancy in the minimum-pressure value is about $0.09 \%$. Minimum pressure computed from all solutions converges monotonically with grid refinement and demonstrates at least a second-order convergence rate.

Figure 34 shows grid convergence of surface pressure near the shock intersection at wing section 4 within the interval $0.2<x / c<0.5$. Most of L4 and L3 solutions completely miss the double-shock structure in this region. Only the FUNFV L3 solution indicates a presence of a shock structure; USM3D and CFL3D L3 solutions miss it. However, all the L1 solutions predict a double-shock structure in this region and agree well with each other. All the L1 solutions predict a pressure plateau between two shocks at $0.3<x / c<0.35$. The normalized $x$-direction grid spacing at this location is $\Delta x / c \approx 0.02$, providing just four grid nodes across the plateau. In spite of the minimal grid resolution, the maximum code-to-code difference between pressure values on this plateau is less than $6 \%$.

Figure 35 shows grid convergence of surface pressure at the most outboard section 7 of the OM6 wing, near the trailing edge in the interval corresponding to $0.98<x / c<1.0$. The $C_{p}$ at this location is close to zero and changes sign; thus, characterization of differences between solutions in terms of relative errors is not insightful. The maximum pressure-coefficient differences within this interval, excluding the trailing edge point, are observed at the left end of the interval. The maximum pressure-coefficient differences between the L4 and L1 grid is less than 0.15 . The difference diminishes with grid refinement; the maximum pressure-coefficient difference between the L2 and L1 grid points is less than 0.03. The maximum code-to-code difference on the L1 grids is less than 0.008. The maximum code-to-code L1 difference at the trailing-edge point is approximately 0.05 . All codes converge monotonically and exhibit a convergence rate that is somewhat less than first order.

Figure 36 shows grid convergence of surface pressure at section 7 , near the minimum-pressure location within the interval $0.025<x / c<0.07$. All codes identify the minimum of pressure at the same location, $x / c \approx 0.041$. Comparing all solutions, coarse-to-fine variation within the interval is about $14 \%$. Note that the FUNFV L4 solution predicts the minimum pressure accurately. The L1 solutions agree well to each other. The code-tocode difference in the minimum-pressure value is about $0.1 \%$. Minimum pressure computed from all solutions converges monotonically with grid refinement; the FUNFV solutions converge from above, the USM3D and CFL3D solutions converge from below. All codes demonstrate at least a second-order convergence rate.

\section{Concluding Remarks}

Detailed grid-convergence studies for two benchmark three-dimensional (3D) flows have been conducted to establish reference solutions for Reynolds-averaged Navier-Stokes (RANS) equations using a "negative" variant of the Spalart-Allmaras turbulence model. The benchmark flows are a subsonic flow around a hemisphere cylinder and a transonic flow around the ONERA M6 wing (OM6) with a sharp trailing edge. The reference solutions have been computed with three widely used CFD codes developed at NASA Langley, FUN3D, USM3D, and CFL3D. The codes use different discretization approaches and iterative solution methods. Two different unstructured-grid second-order node-centered discretizations available in FUN3D are used for the hemisphere-cylinder computations: the FUNFV discretization uses a standard finite-volume scheme and the SFE discretization uses a recently added stabilized finite-element formulation. SFE is not used for OM6 computations. USM3D uses an unstructured-grid second-order cell-centered finite-volume formulation. CFL3D uses a second-order cell-centered structured-grid formulation. Five families of consistently-refined nested grids of different topology have been generated for the studies, including both structured grids and unstructured grids with various types of elements. The finest family grids provide from 60 million to over 400 million degrees of freedom.

To eliminate iterative errors, all solutions on all grids have converged to near machine-zero residual levels. Although turbulence model validation is not the focus of the paper, the reference solutions have been compared with available experimental data. The main thrust of the paper is assessing variation between CFD solutions computed with different codes on different families of consistently-refined grids. The assessment includes two characteristics: (1) relative coarse-to-fine variation of solutions computed on grids of different families and (2) relative code-to-code variation of solutions computed on the finest family grids. 
All codes show close agreement in predicting aerodynamic coefficients for the separated flow around the hemisphere-cylinder configuration. The code-to-code discrepancy among all aerodynamic coefficients computed on the finest family grids is less than $4.5 \%$ and variation among a core group of four solutions is less than $0.75 \%$. The coefficients appear to converge to the same limit with grid refinement, but no convergence order can be discerned for the observed convergence. There is more uncertainty about the grid convergence limit of the maximum eddy viscosity.

The surface pressure and skin friction in different fine-grid solutions overplot in most global views. A local disagreement among the codes of about $15 \%$ is observed in the vicinity of the leeside just past the middle section of the cylinder. This disagreement is limited to skin friction and is not reflected in surface pressure. Various off-body solution components probed outside of this region also overplot. Local solution characteristics, such as surface pressure minima and the circumferential angle of vortex separation and reattachment locations, also converge to the same limit with grid refinement. The reference solutions compare well with available experimental data.

The reference solutions for a transonic flow around OM6 have been computed using the three formulations, FUNFV, USM3D, and CFL3D. The aerodynamic coefficients computed by different codes on the finest grids of different families agree well; the maximum difference among all coefficients does not exceed $0.73 \%$. In spite of a close agreement between fine-grid solutions, no asymptotic convergence order can be established. The difference in maximum eddy viscosity is $10.3 \%$, which is much larger than the corresponding difference in the aerodynamic coefficients.

The surface pressure computed with the three codes have been compared at seven OM6 wing sections. The pressure profiles computed on the finest grids overplot in the global views. Away from shocks, all the pressure profiles computed on the two finest grids are close to each other, within a 1-2\% range. Increased grid resolution allows for an improved resolution of the lambda-shock feature that was a challenge in past OM6 computations. As compared to previous studies available in the literature, the present solutions on the finest grids provide an improved agreement with the experiment.

\section{Acknowledgments}

The NASA Aeronautics Research Mission Directorate Transformative Aeronautics Concepts Program Transformative Tools and Technologies project and Airspace Operations and Safety Program Airspace Technology Demonstrations project funded the work reported herein. All computations were performed on the NASA Langley Research Center K cluster and the NASA Advanced Supercomputing facility's Pleiades supercomputer.

\section{References}

1 "Turbulence Modeling Resource Website," http://turbmodels.larc.nasa.gov, last accessed November 15, 2017.

${ }^{2}$ Rumsey, C. L., Smith, B. R., and Huang, G. P., "Description of a Website Resource for Turbulence Modeling Verification and Validation," AIAA Paper 2010-4742, 2010.

${ }^{3}$ Rumsey, C. L., "Recent Developments on the Turbulence Modeling Resource Website," AIAA Paper 2015-2927, 2015.

${ }^{4}$ Brown, D. A., Buckley, H., Osusky, M., and Zingg, D. W., "Performance of a Newton-Krylov-Schur Algorithm for the Numerical Solution of the Steady Reynolds-Averaged Navier-Stokes Equations (Invited)," AIAA Paper 2015-1744, 2015.

${ }^{5}$ Gleize, V., Dumont, A., Mayeur, J., and Destarac, D., "RANS simulations on TMR test cases and M6 wing with the ONERA elsA flow solver (Invited)," AIAA Paper 2015-1745, 2015.

${ }^{6}$ Diskin, B., Thomas, J., Rumsey, C. L., and Schwöppe, A., "Grid Convergence for Turbulent Flows (Invited)," AIAA Paper 2015-1746, 2015.

${ }^{7}$ Pandya, M. J., Diskin, B., Thomas, J., and Frink, N. T., "Improved Convergence and Robustness of USM3D Solutions on Mixed Element Grids (Invited)," AIAA Paper 2015-1747, 2015.

${ }^{8}$ Brazell, M. J. and Mavriplis, D. J., "High-Order Discontinuous Galerkin Mesh Resolved Turbulent Flow Simulations of a NACA 0012 Airfoil (Invited)," AIAA Paper 2015-1748, 2015.

${ }^{9} \mathrm{Hu}$, Y., Wagner, C., Allmaras, S., Galbraith, M., and Darmofal, D. L., "Application of a Higher-order Adaptive Method to RANS Test Cases (Invited)," AIAA Paper 2015-1530, 2015.

${ }^{10}$ Anderson, W. K., Ahrabi, B. R., and Newman, J., "Finite-Element Solutions for Turbulent Flow over the NACA 0012 Airfoil (Invited)," AIAA Paper 2015-1531, 2015.

${ }^{11}$ Fidkowski, K., "High-Order Output-Based Adaptive Simulations of Turbulent Flow in Two Dimensions (Invited)," AIAA Paper 2015-1532, 2015.

12 Diskin, B., Thomas, J., Rumsey, C. L., and Pandya, M. J., "Reference Solutions for Benchmark Three Dimensional Turbulent Flows," AIAA Paper 2016-0858, 2016.

${ }^{13}$ Park, M. A. and Anderson, W. K., "Spatial Convergence of Three Dimensional Turbulent Flows," AIAA Paper 2016-0859, 2016.

12 of 35 
${ }^{14}$ Pandya, M. J., Diskin, B., Thomas, J., and Frink, N. T., "Assessment of Preconditioner for a USM3D Hierarchical Adaptive Nonlinear Iteration Method (HANIM) (Invited)," AIAA Paper 2016-0860, 2016.

15 Brazell, M. J., Ahrabi, B. R., and Mavriplis, D. J., "Discontinuous Galerkin Turbulent Flow Simulations of NASA Turbulence Model Validation Cases and High Lift Prediction Workshop Test Case DLR-F11," AIAA Paper 2016-0861, 2016.

${ }^{16}$ Fidkowski, K. and Ceze, M., "High-Order Output-Based Adaptive Simulations of Turbulent Flow in Three Dimensions (Invited)," AIAA Paper 2016-0862, 2016.

${ }^{17}$ Mayeur, J., Dumont, A., Gleize, V., and Destarac, D., "RANS simulations on TMR 3D test cases with the ONERA elsA flow solver," AIAA Paper 2016-1357, 2016.

${ }^{18}$ Hashimoto, A., Ishida, T., Aoyama, T., Takekawa, K., and Hayashi, K., "Results of Three-Dimensional Turbulent Flow with FaSTAR," AIAA Paper 2016-1358, 2016.

${ }^{19}$ Yanagita, Y., Tong, O., and Katz, A. J., "Critical Evaluation of Turbulence Modeling with the Flux Correction Method on Strand Grids," AIAA Paper 2016-1360, 2016.

${ }^{20}$ Erwin, J. T. and Glasby, R. S., "Application of HPCMP CREATE ${ }^{T} M-A V$ COFFE for Three-Dimensional Turbulent Flow Cases," AIAA Paper 2016-1361, 2016.

${ }^{21}$ Spalart, P. R. and Allmaras, S. R., "A One-Equation Turbulence Model for Aerodynamic Flows," Recherche Aerospatiale, No. 1, 1994, pp. 5-21.

${ }^{22}$ Allmaras, S. R., Johnson, F. T., and Spalart, P. R., "Modifications and Clarifications for the Implementation of the SpalartAllmaras Turbulence Model," Proc. of Seventh International Conference on Computational Fluid Dynamics, ICCFD7-1902, Big Island, Hawaii, 2012.

23 "Special Section on Evaluation of RANS Solvers on Benchmark Aerodynamics Flows," AIAA Journal, 2016, Vol. 54, No. 9, pp. 2561-2704.

${ }^{24}$ AIAA, "Guide for the Verification and Validation of Computational Fluid Dynamics Simulations," AIAA G-077-1998, 1998.

${ }^{25}$ Oberkampf, W. L. and Roy, C. J., Verification and Validation in Scientific Computing, Cambridge University Press, 2010.

${ }^{26}$ Nishikawa, H. and Diskin, B., "Customized Grid Generation and Processing for Benchmark Three-Dimensional Flows," SciTech-2018, Kissimmee, FL, Jan., 2018, To be published as AIAA Paper.

${ }^{27}$ Biedron, R. T., Carlson, J.-R., Derlaga, J. M., Gnoffo, P. A., Hammond, D. P., Jones, W. T., Kleb, B., Lee-Rausch, E. M., Nielsen, E. J., Park, M. A., Rumsey, C. L., Thomas, J. L., and Wood, W. A., FUN3D Manual: 13.2, NASA-TM-2017-219661, September 2017.

${ }^{28}$ Abdol-Hamid, K. S., Carlson, J.-R., Rumsey, C. L., Lee-Rausch, E. M., and Park, M. A., "DPW-6 Results Using FUN3D With Focus on k-kL-MEAH2015 Turbulence Model," AIAA Paper 2017-0962, 2017.

${ }^{29}$ Chwalowski, P. and Heeg, J., "FUN3D Analyses in Support of the Second Aeroelastic Prediction Workshop," AIAA Paper 2016-3122, 2016.

${ }^{30}$ Silva, W. A., Sanetrik, M. D., and Chwalowski, P., "Using FUN3D for Aeroelatic, Sonic Boom, and AeroPropulsoServoElastic (APSE) Analyses of a Supersonic Configuration," AIAA Paper 2016-1319, 2016.

31 Jain, R., Biedron, R. T., Jones, W., and Lee-Rausch, E. M., "Modularization and Validation of NASA FUN3D as a HPCMP CREATE-AV Helios Near-body Solver," AIAA Paper 2016-1298, 2016.

32 Vatsa, V. N., Khorrami, M. R., Rhoads, J., and Lockard, D. P., "Aeroacoustic Simulations of a Nose Landing Gear using FUN3D on Pointwise Unstructured Grids," AIAA Paper 2015-3255, 2015.

${ }^{33}$ Anderson, W. K., Newman, J. C., and Karman, S. L., "Stabilized Finite Elements in FUN3D," AIAA Paper 2017-0077, 2017.

${ }^{34}$ Anderson, W. K., Thomas, J. L., and van Leer, B., "Comparison of Finite Volume Flux Vector Splittings for the Euler Equations," AIAA Journal, Vol. 24, 1986, pp. 1453-1460.

${ }^{35}$ Frink, N. T., Pirzadeh, S. Z., Parikh, P. C., Pandya, M. J., and M. K. Bhat, M., "The NASA Tetrahedral Unstructured Software System (TetrUSS)," The Aeronautical Journal, Vol. 104, No. 1040, 2000, pp. 491-499.

${ }^{36}$ Pandya, M. J., Frink, N. T., Abdol-Hamid, K. S., Samareh, J., Parlette, E. B., and Taft, J., "Enhancements to TetrUSS for NASA Constellation Program," Journal of Spacecraft and Rockets, Vol. 49, No. 4, 2012, pp. 617-631.

${ }^{37}$ Bauer, S. X., Krist, S. E., and Compton, W. B., "Generation of the Ares I-X Flight Test Vehicle Aerodynamic Data Book and Comparison To Flight," AIAA Paper 2011-0011, 2011.

38 Abdol-Hamid, K. S., Chaffari, F., and Parlette, E. B., "Ares I Vehicle Computed Turbulent Ascent Aerodynamic Data Development and Analysis," Journal of Spacecraft and Rockets, Vol. 49, No. 4, 2012, pp. 596-608.

${ }^{39}$ Elmiligui, A., Abdol-Hamid, K. S., Cavallo, P. A., and Parlette, E. B., "USM3D Simulations for the F-16XL Aircraft Configuration," Journal of Aircraft, 2017, Publication Date (online): April 04, 2017.

${ }^{40}$ Green, B. E., "Computational Prediction of Nose-Down Control for F/A-18E at High Alpha," Journal of Aircraft, Vol. 45, No. 5, 2008, pp. 1661-1668.

${ }^{41}$ Hooker, J. R., Wick, A., Zeune, C. H., and Agelastos, A., "Design and Transonic Wind Tunnel Testing of a Cruise Efficient STOL Military Transport," AIAA Paper 2013-2920, 2013.

42 Pandya, M. J., Diskin, B., Thomas, J. L., and Frink, N. T., "Improved Convergence and Robustness of USM3D Solutions on Mixed-Element Grids," AIAA Journal, Vol. 54, No. 9, 2016, pp. 2589-2610, Also AIAA Paper 2015-1747.

${ }^{43}$ Pandya, M. J., Diskin, B., Thomas, J. L., and Frink, N. T., "Assessment of USM3D Hierarchical Adaptive Nonlinear Method Preconditioners for Three-Dimensional Cases," AIAA Journal, Vol. 55, No. 10, 2017, pp. 3409-3424, Also AIAA Paper $2016-0860$.

${ }^{44}$ Courant, R. and Hilbert, D., Methods of Mathematical Physics, Interscience, New York, 1953.

${ }^{45}$ Holmes, D. G. and Connell, S. D., "Solution of the 2D Navier-Stokes equations on unstructured adaptive grids," AIAA Paper 89-1932, 1989.

${ }^{46}$ Rausch, R. D., Batina, J. T., and Yang, H. T., "Spatial adaptation procedures on unstructured meshes for accurate unsteady aerodynamic flow computation," AIAA J., Vol. 30, No. 5, April 1992, pp. 1243-1251.

${ }^{47}$ Frink, N. T., "Assesment of an unstructured-grid method for predicting 3-D turbulent viscous flows," AIAA Paper 96-0292, 1996.

13 of 35 
48 Mitchell, C. R., "Improved Reconstruction Schemes for the Navier-Stokes Equations on Unstructured Meshes," AIAA Paper 1994-0642, 1994.

${ }^{49}$ Frink, N. T., "Tetrahedral Unstructured Navier-Stokes Method for Turbulent Flows," AIAA J., Vol. 36, No. 11, November 1998, pp. 1975-1982.

50 Tinoco, E. N., Brodersen, O., Keye, S., and Laflin, K., "Summary of Data from the Sixth AIAA CFD Drag Prediction Workshop: CRM Cases 2 to 5," AIAA Paper 2017-1208, 2017.

${ }^{51}$ Rumsey, C. L. and Slotnick, J. P., "Overview and Summary of the Second AIAA High-Lift Prediction Workshop," Journal of Aircraft, Vol. 52, No. 4, 2015, pp. 1006-1025.

${ }^{52}$ Abdol-Hamid, K. S., Carlson, J.-R., and Rumsey, C. L., "Verification and Validation of the k-kL Turbulence Model in FUN3D and CFL3D Codes," AIAA Paper 2016-3941, 2016.

${ }^{53}$ Diskin, B., Thomas, J. L., Rumsey, C., and Schwöppe, A., "Grid Convergence of Reynolds-Averaged Navier-Stokes Solutions for Benchmark Flows in Two Dimensions," AIAA J., Vol. 54, No. 9, September 2016, pp. 2563-2588.

54 Tsieh, T., "An Investigation of Separated Flow About a Hemisphere-Cylinder at 0- to 19-Deg Incidence in the Mach Number Range from 0.6 to 1.5," AEDC-TR-76-112, 1976.

${ }^{55}$ Diskin, B. and Thomas, J. L., "Comparison of Node-Centered and Cell-Centered Unstructured Finite-Volume Discretizations: Inviscid Fluxes," AIAA J., Vol. 49, No. 4, 2011, pp. 836-854.

${ }^{56}$ Diskin, B. and Thomas, J. L., "Erratum: Comparison of Node-Centered and Cell-Centered Unstructured Finite-Volume Discretizations: Inviscid Fluxes," AIAA J., Vol. 51, No. 1, 2013, pp. 277.

${ }^{57}$ Schmitt, V. and Charpin, F., "Pressure Distribution on the ONERA-M6-Wing at Transonic Mach Numbers," In Experimental Data Base for Computer Program Assessment. Report of the Fluid Dynamics Panel Working Group 04, AGARD AR $138,1979$. 


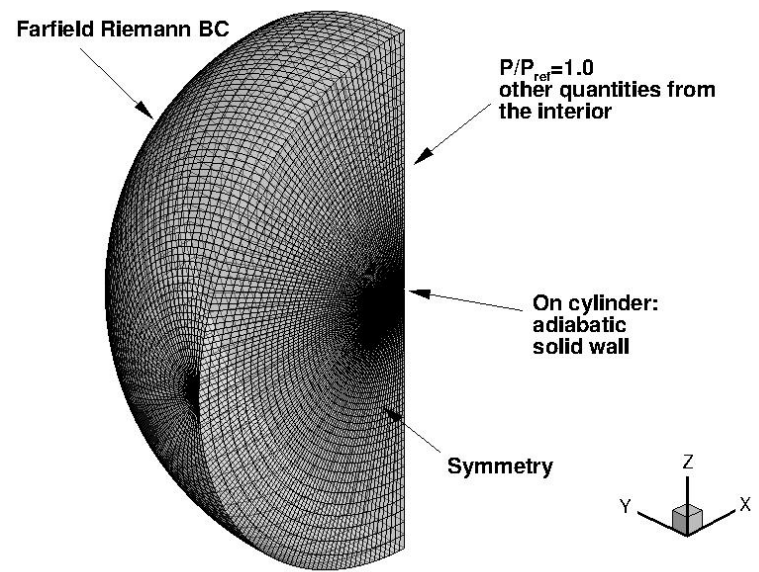

Figure 1: Global view of hemisphere cylinder geometry and boundary conditions.

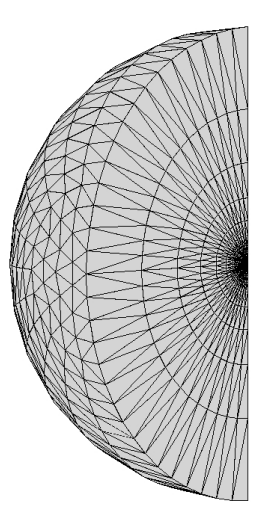

(a) Farfield/symmetry boundary

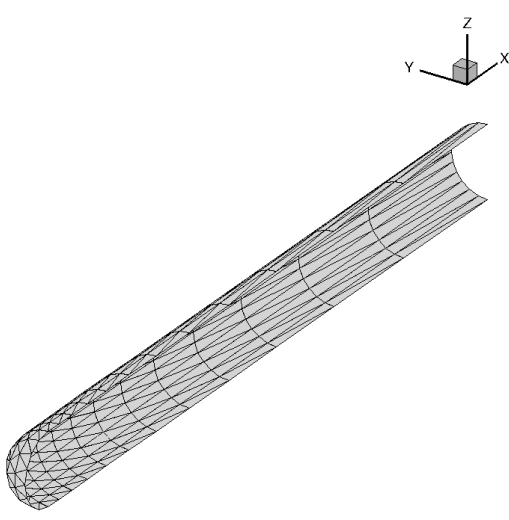

(b) Surface grid

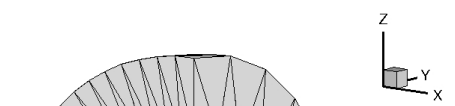

(c) Outflow/symmetry boundary

Figure 2: Hemisphere cylinder, family 2 (tet) grid.

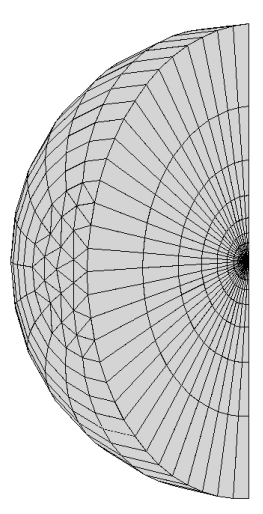

(a) Farfield/symmetry boundary

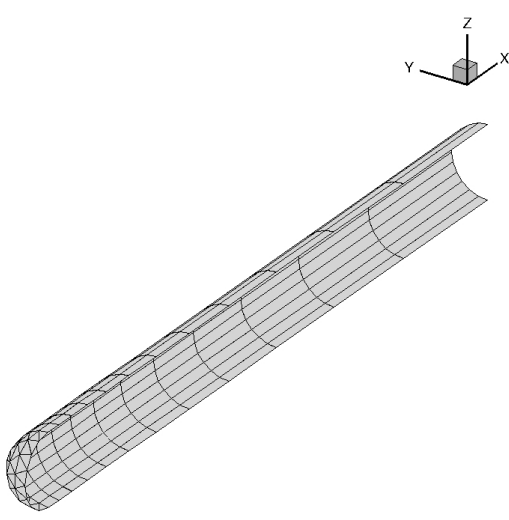

(b) Surface grid

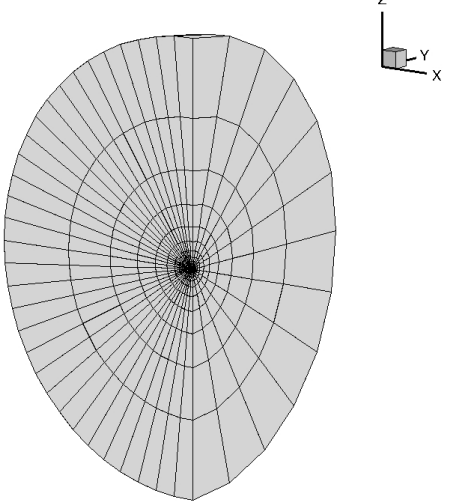

(c) Outflow/symmetry boundary

Figure 3: Hemisphere cylinder, family 4 (prism/hex) grid. 


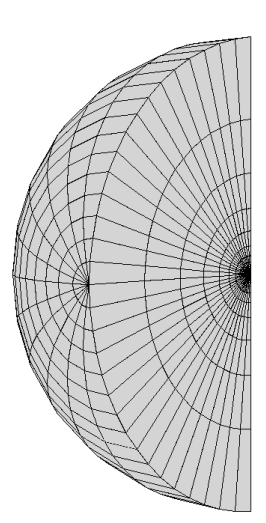

(a) Farfield/symmetry boundary

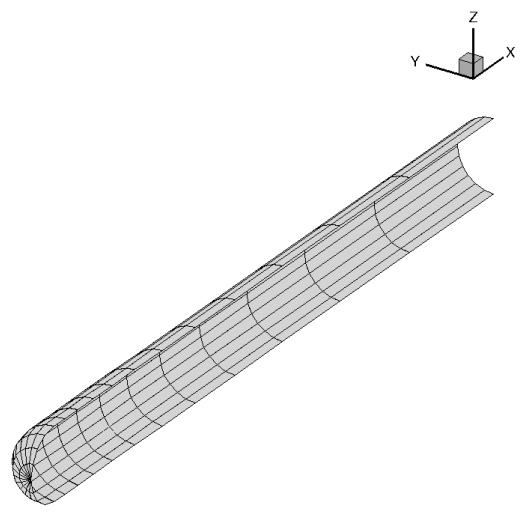

(b) Surface grid

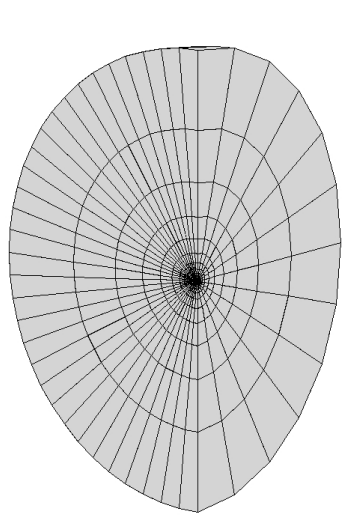

$L_{X}^{Z}$

Figure 4: Hemisphere cylinder, family 5 (struct) grid.

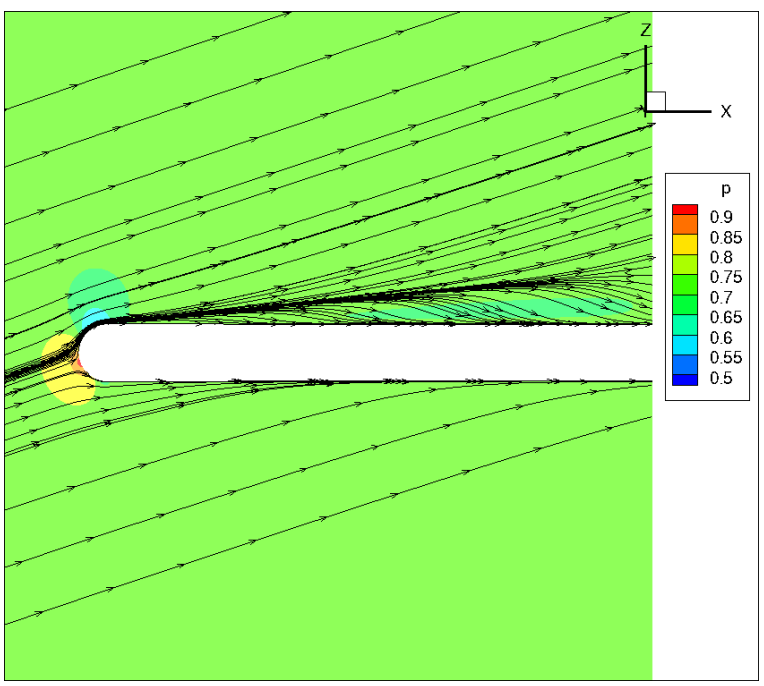

(a) $y=0$

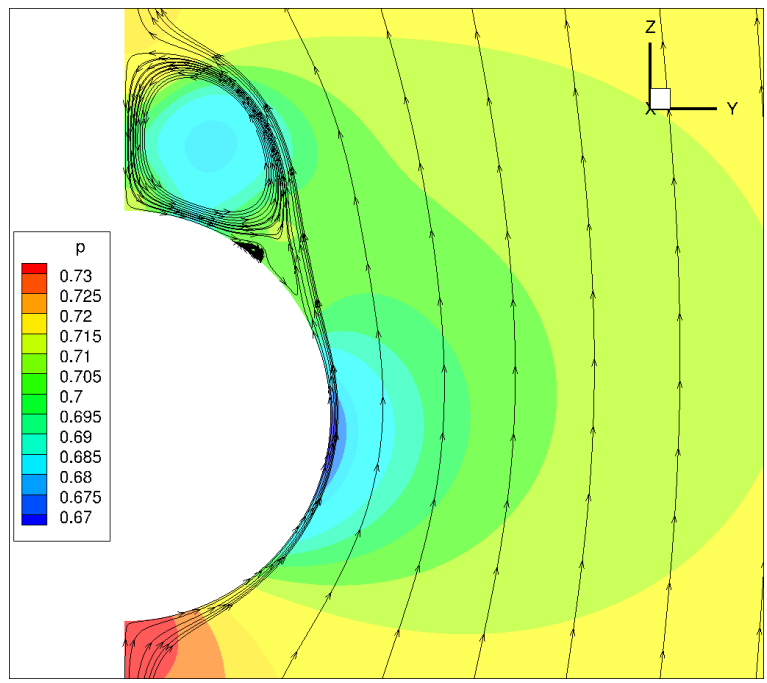

(c) $x=5$

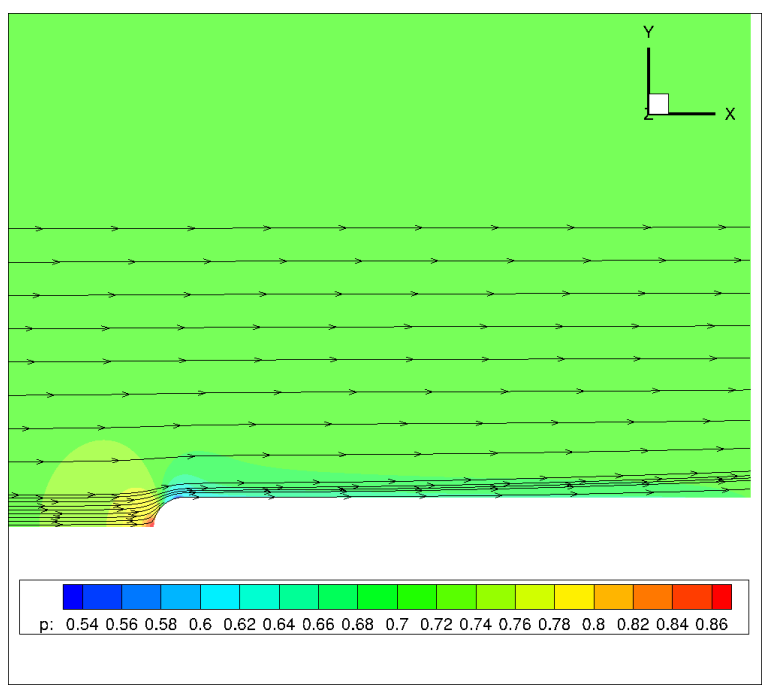

(b) $z=0$

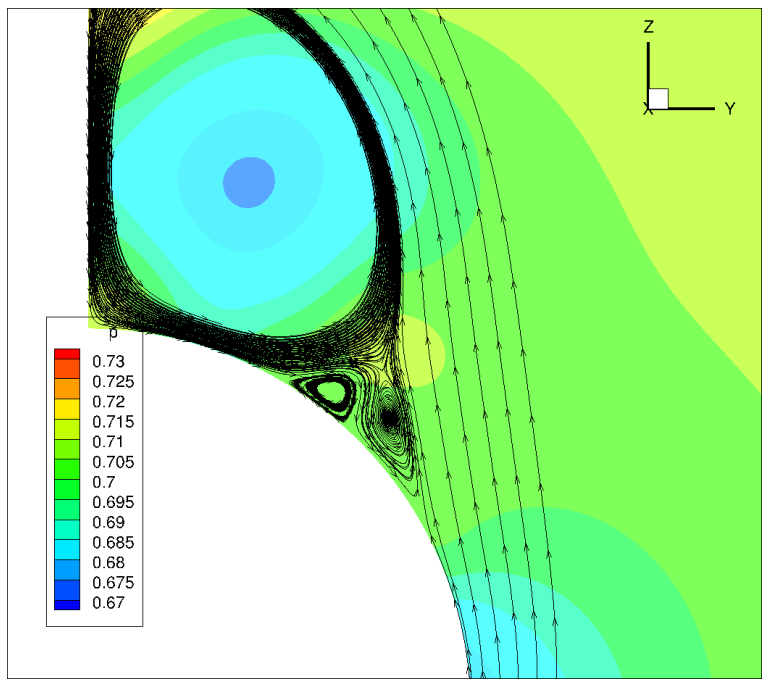

(d) $x=6$

Figure 5: Hemisphere cylinder: global view of FUNFV solution computed on prism/hex L1 grid of family 4 . 


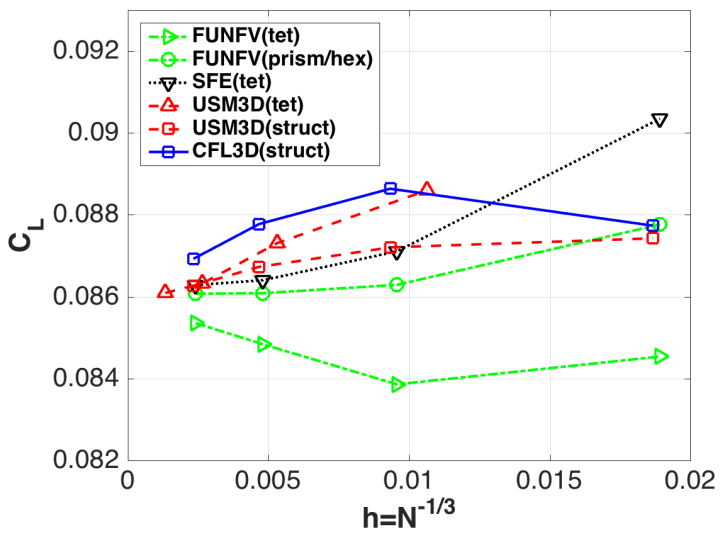

(a) Lift

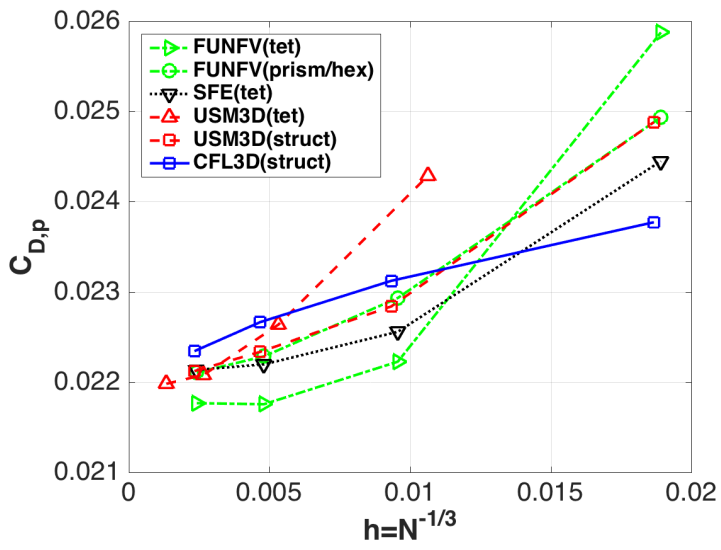

(c) Pressure drag

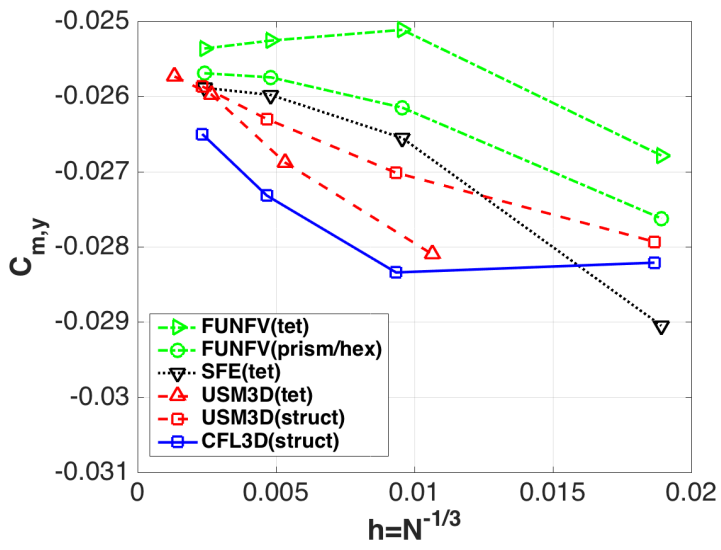

(e) Pitching moment

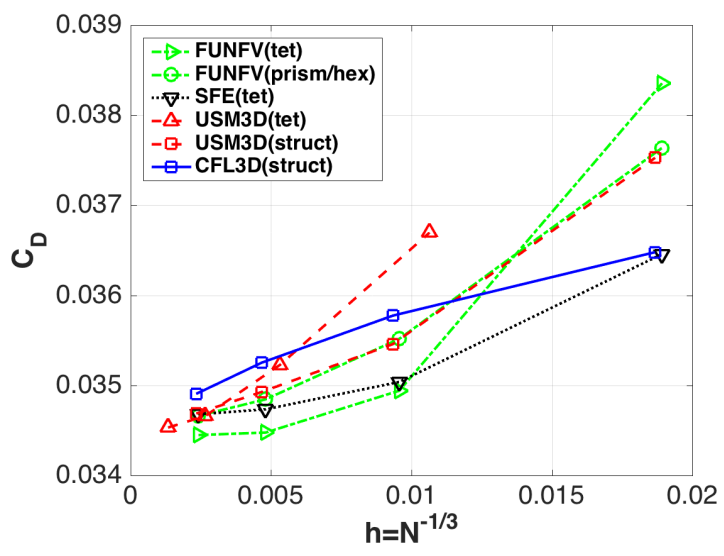

(b) Total drag

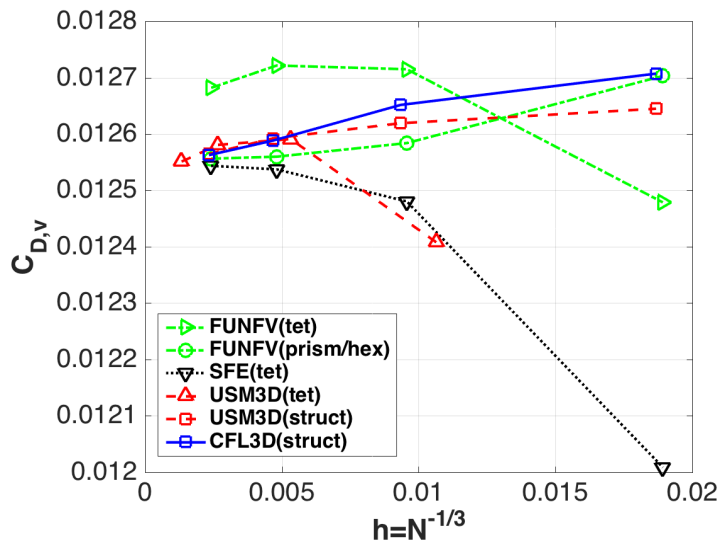

(d) Viscous drag

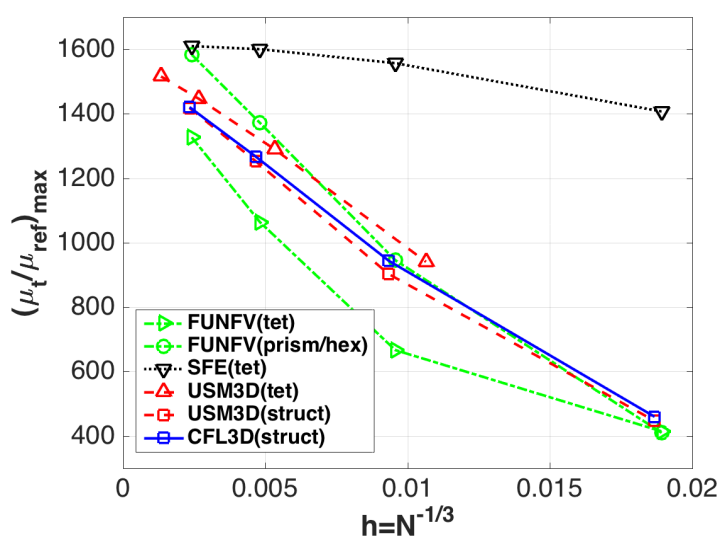

(f) Maximum eddy viscosity

Figure 6: Hemisphere cylinder: grid convergence of aerodynamic forces, pitching moment, and maximum eddy viscosity. 


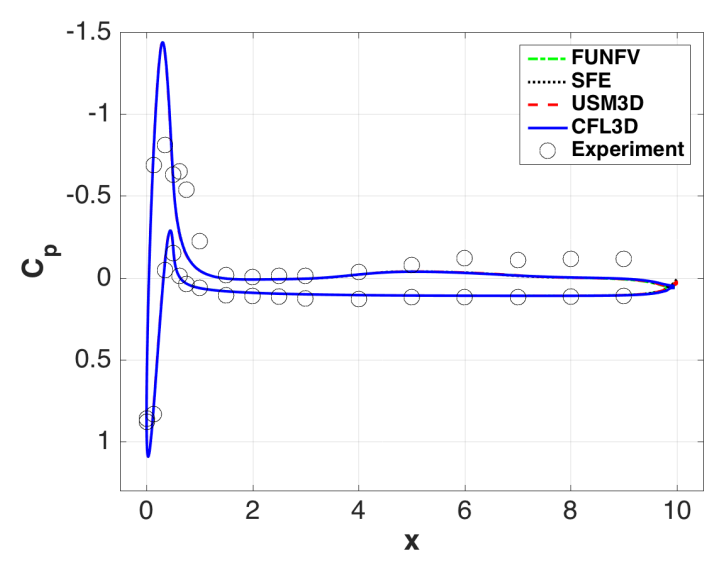

(a) Surface pressure

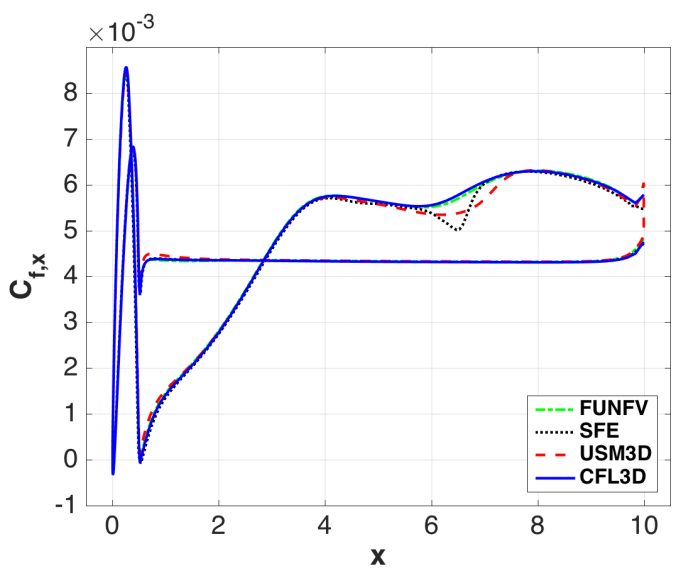

(b) Skin friction, $\mathrm{x}$ component

Figure 7: Hemisphere cylinder: global view of surface pressure and skin friction at symmetry plane $(y=0)$.

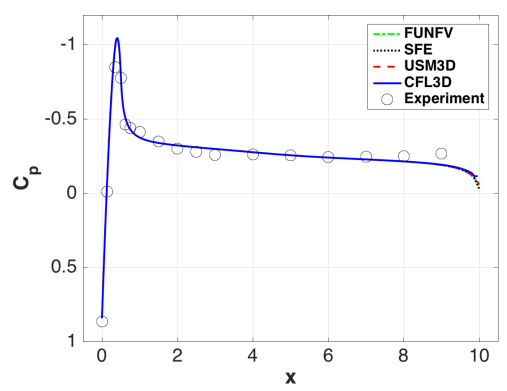

(a) Surface pressure

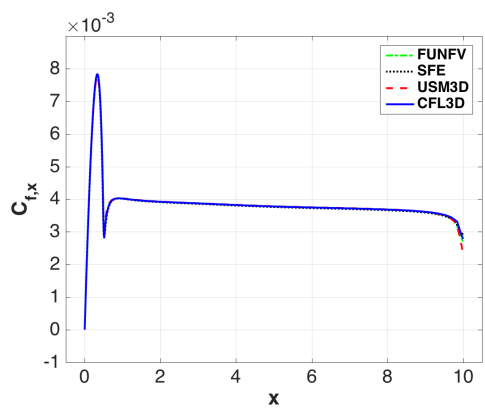

(b) Skin friction, $\mathrm{x}$ component

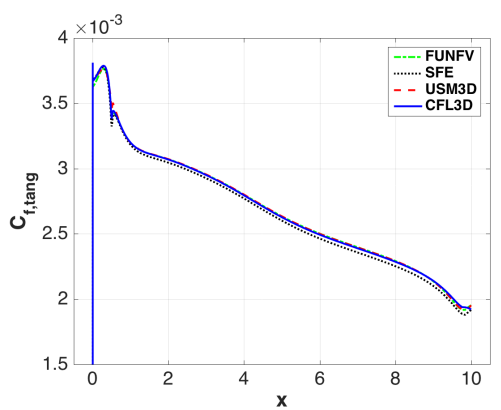

(c) Skin friction, tangential component

Figure 8: Hemisphere cylinder: global view of surface pressure and skin friction at $z=0$ plane.

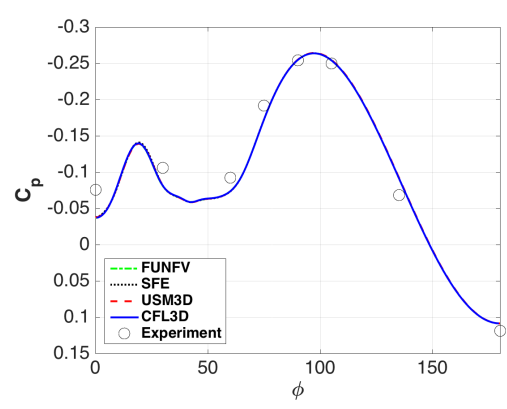

(a) Surface pressure

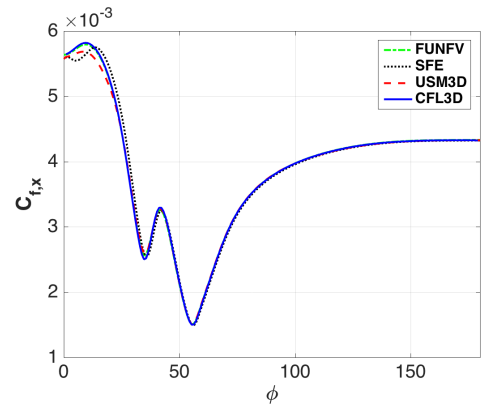

(b) Skin friction, x component

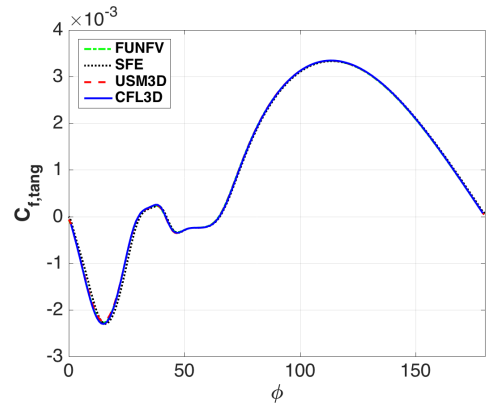

(c) Skin friction, tangential component

Figure 9: Hemisphere cylinder: global view of circumferential variation of surface pressure and skin friction at $x=5.0$ plane. 


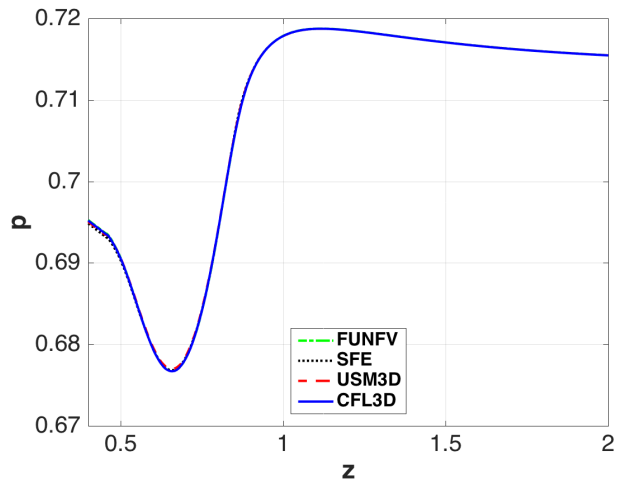

(a) Pressure

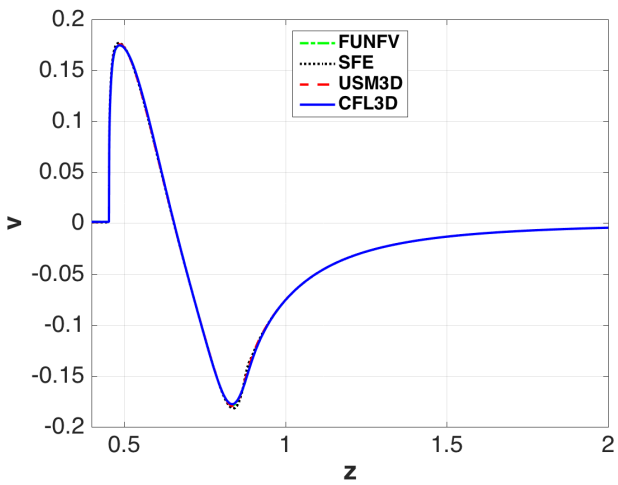

(c) $V$-velocity

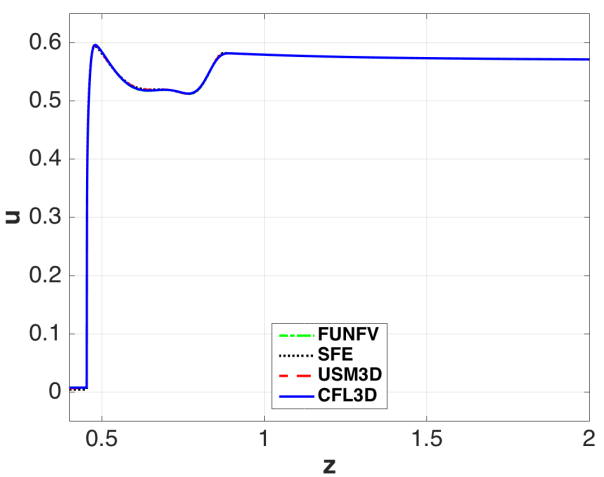

(b) $U$-velocity

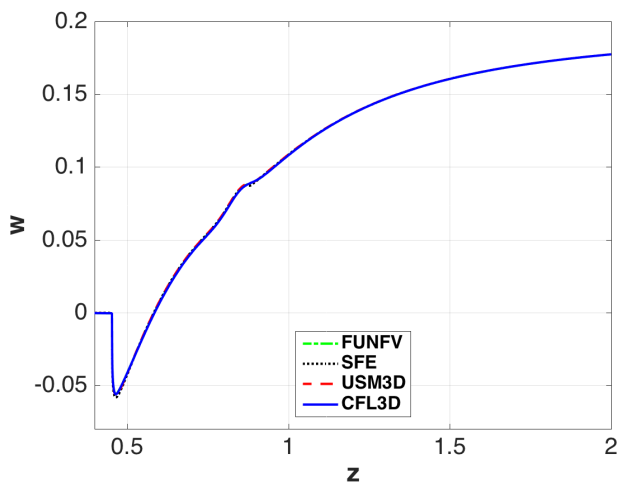

(d) $W$-velocity

Figure 10: Hemisphere cylinder: global view of grid convergence of off-body variable near the upper surface along vertical line corresponding to $x=5.0$ and $y=0.21$. 


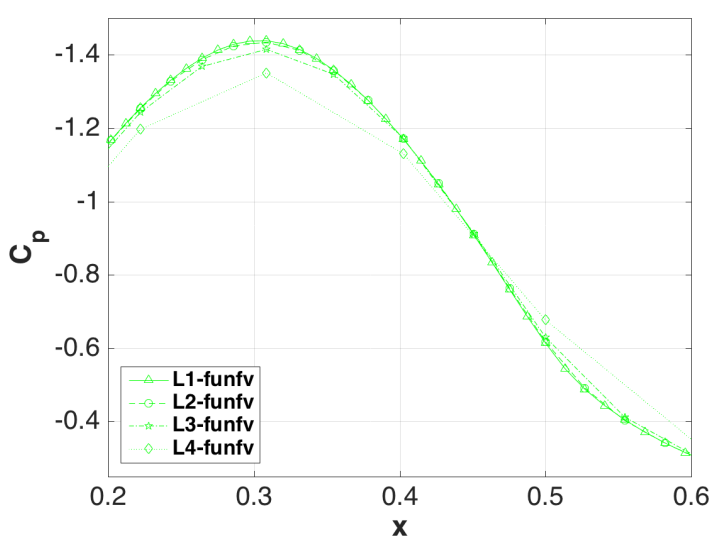

(a) FUNFV

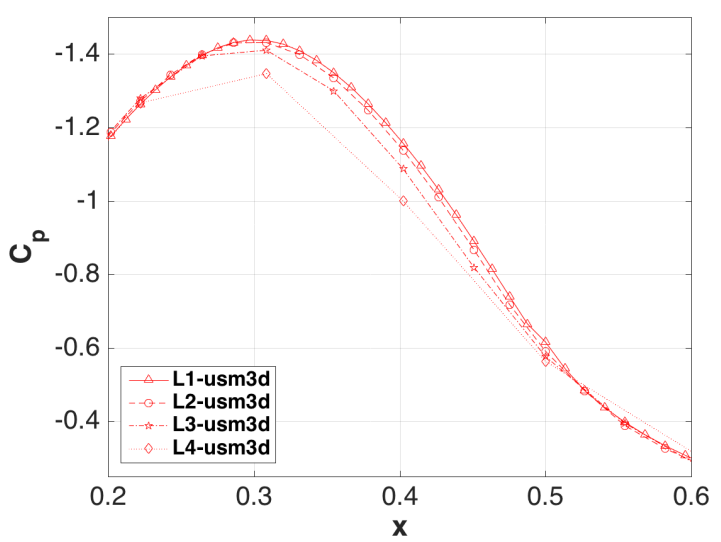

(c) USM3D

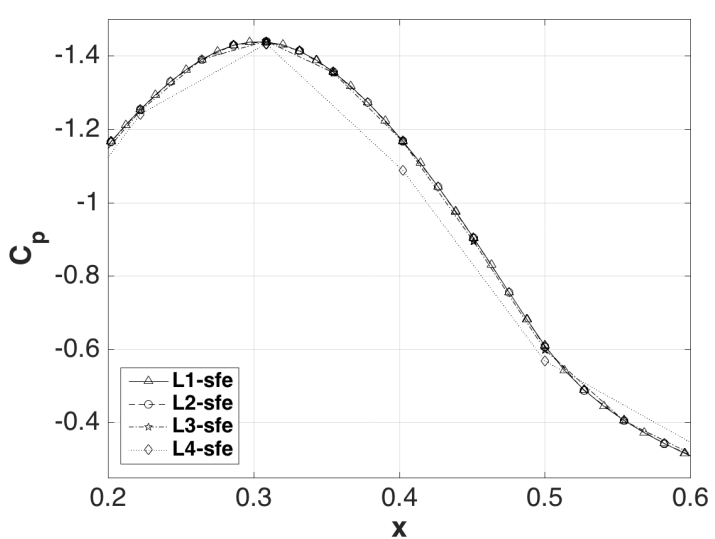

(b) SFE

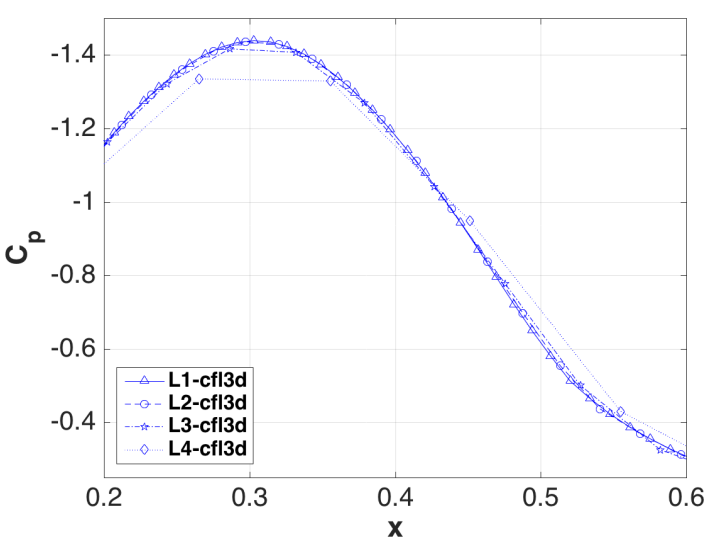

(d) CFL3D

Figure 11: Hemisphere cylinder zoomed view: grid convergence of surface pressure near leeside minimum.

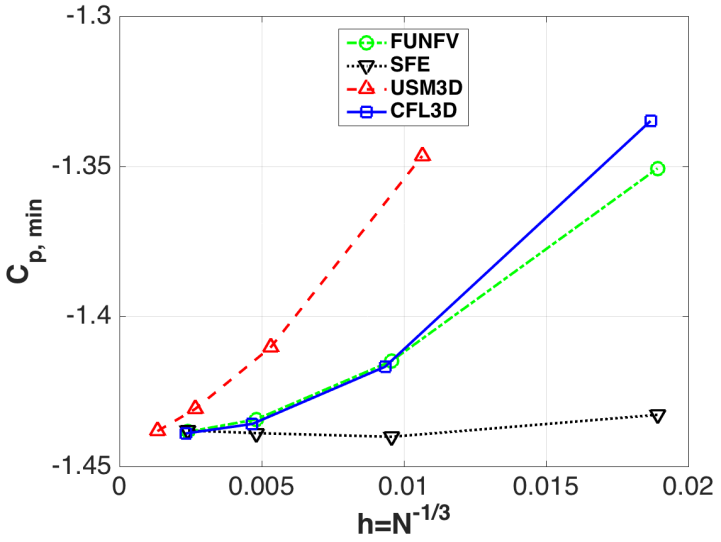

(a) Leeside

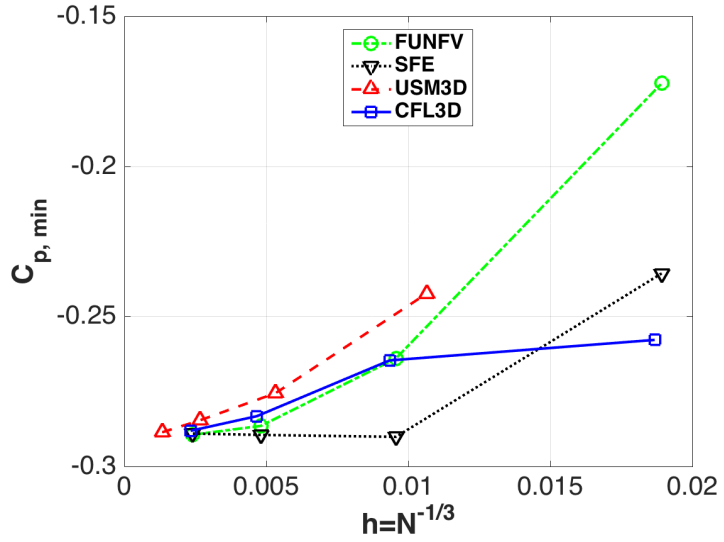

(b) Windward side

Figure 12: Hemisphere cylinder: grid convergence of minimum surface pressure at $y=0$ plane. 


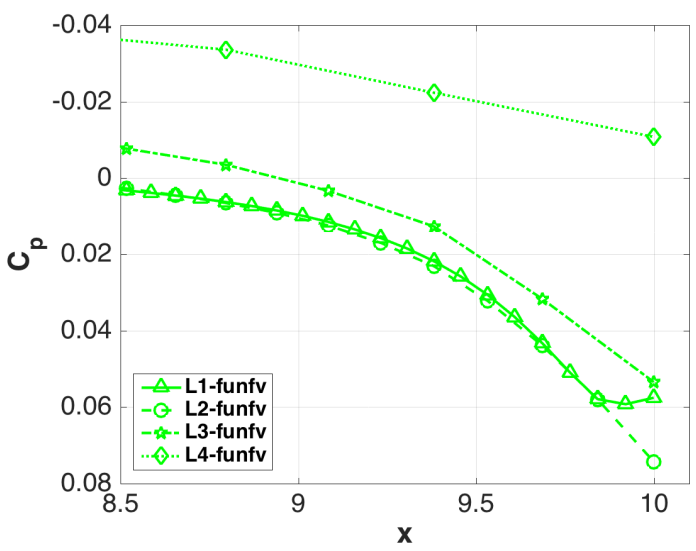

(a) FUNFV

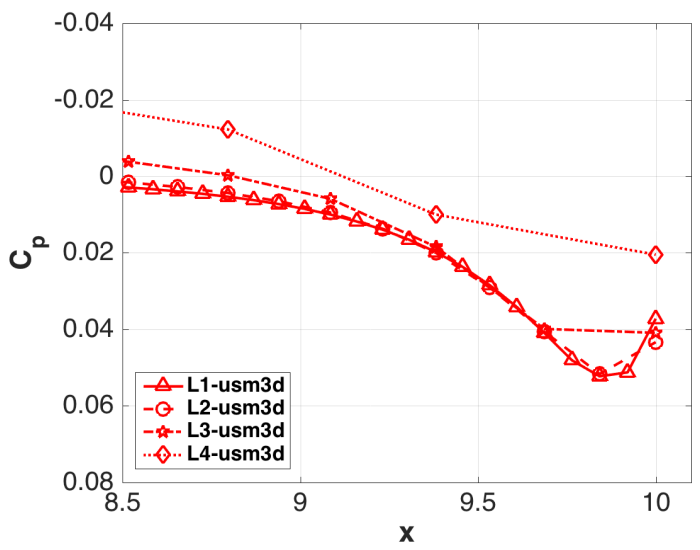

(c) USM3D

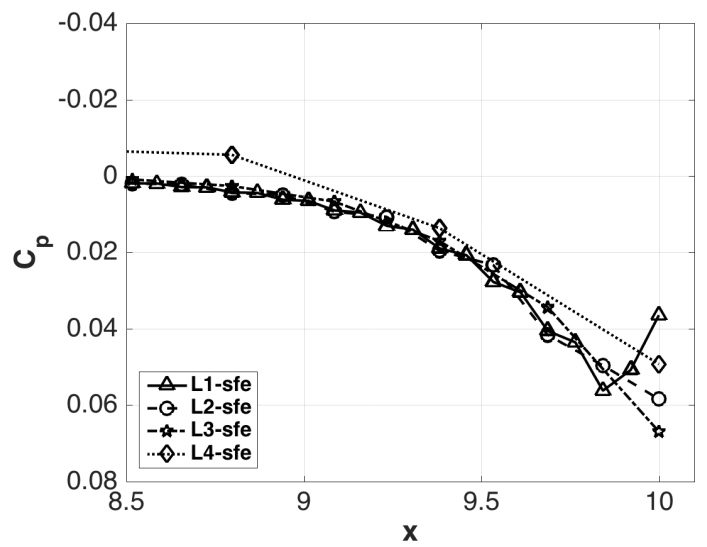

(b) SFE

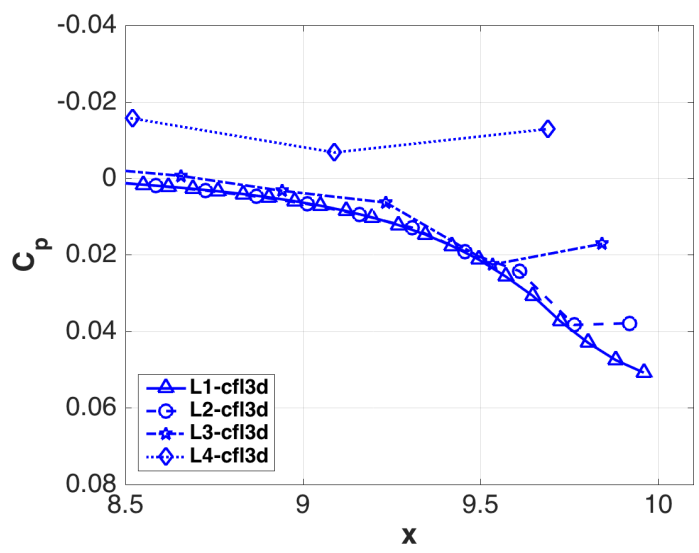

(d) CFL3D

Figure 13: Hemisphere cylinder zoomed view: grid convergence of surface pressure near leeside outflow.

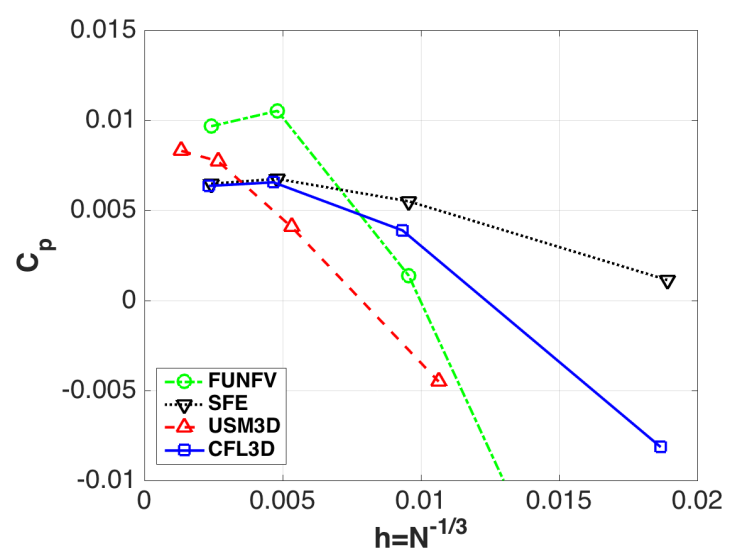

(a) $\mathrm{x}=9$

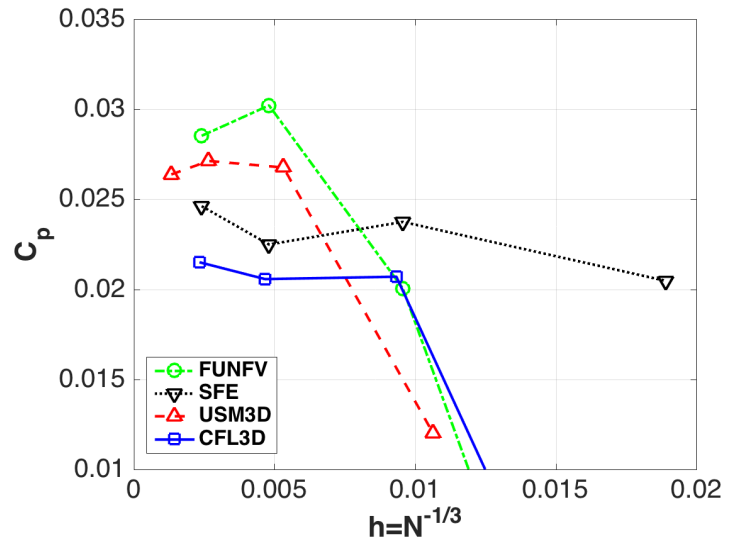

(b) $\mathrm{x}=9.5$

Figure 14: Hemisphere cylinder: grid convergence of leeside surface pressure near outflow boundary. 


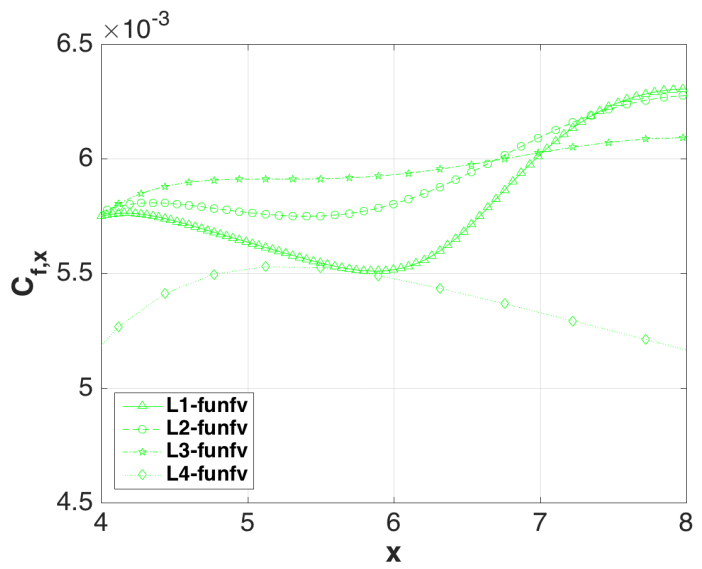

(a) FUNFV

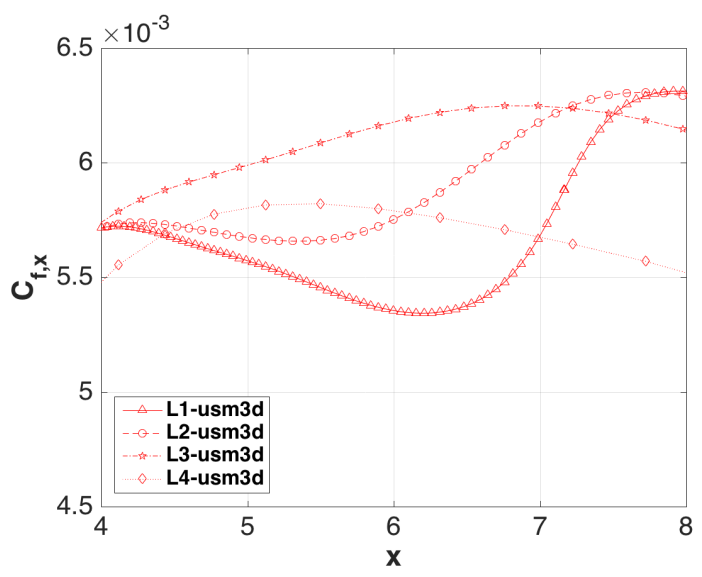

(c) USM3D

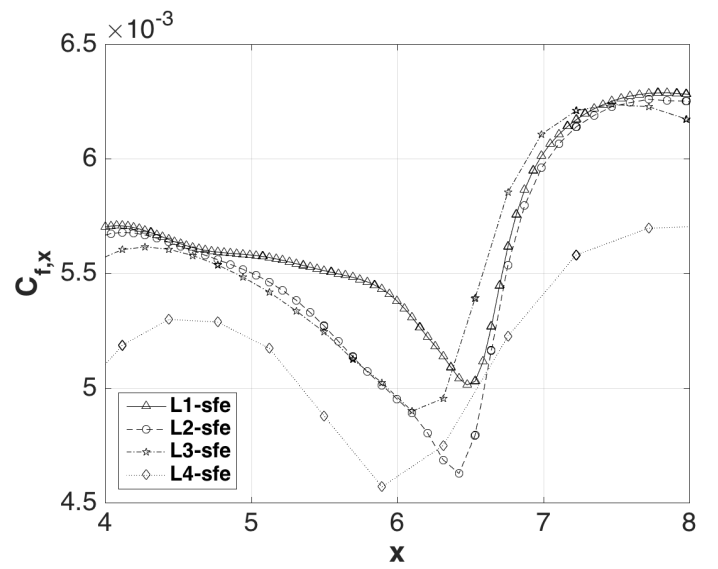

(b) SFE

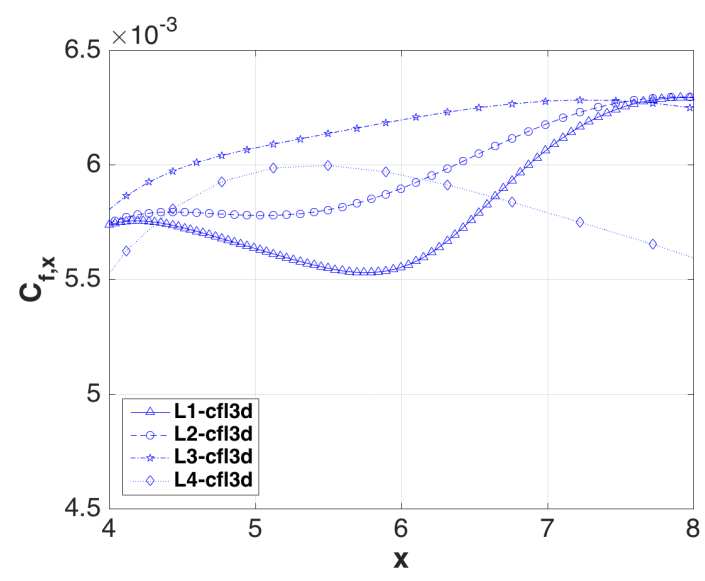

(d) CFL3D

Figure 15: Hemisphere cylinder zoomed view: grid convergence of leeside skin friction near $x=6.0$. 


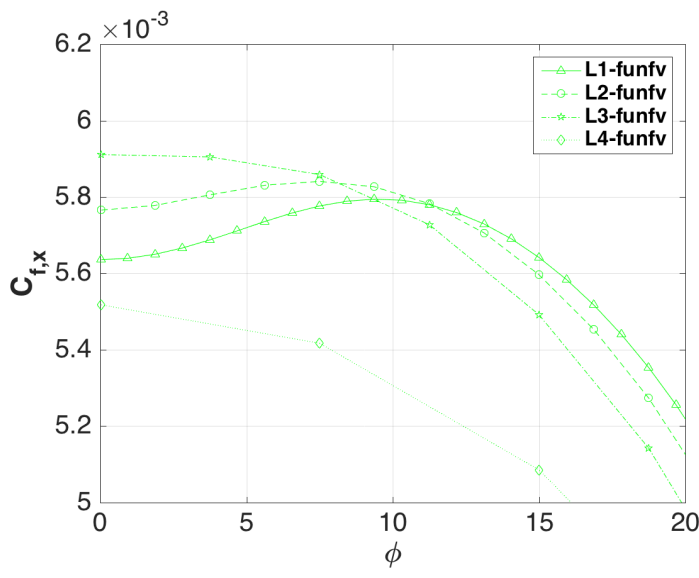

(a) FUNFV

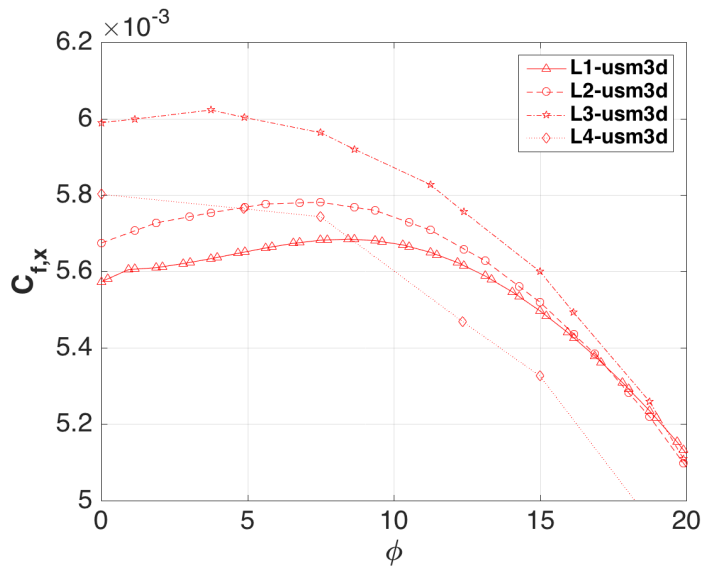

(c) USM3D

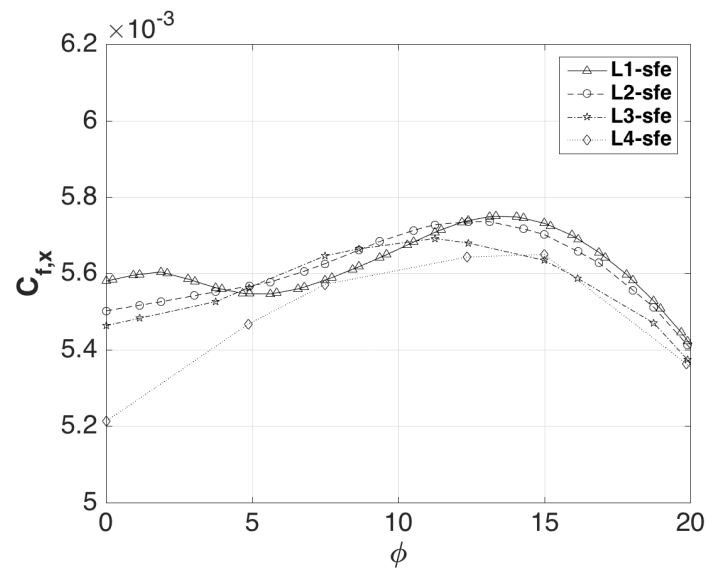

(b) SFE

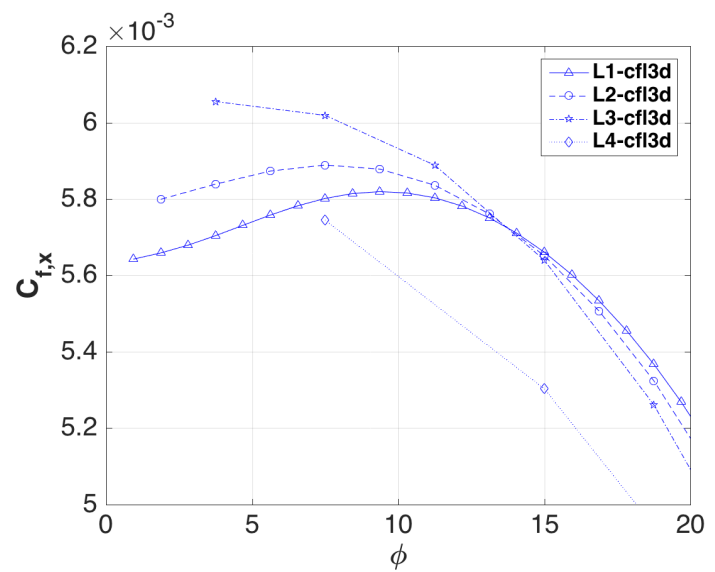

(d) CFL3D

Figure 16: Hemisphere cylinder zoomed view: grid convergence of $x$-component of skin friction in $x=5$ plane near leeside. 


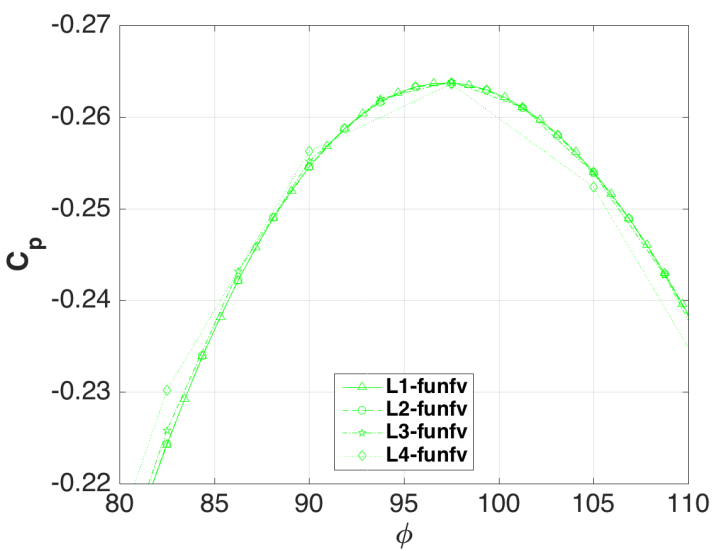

(a) FUNFV

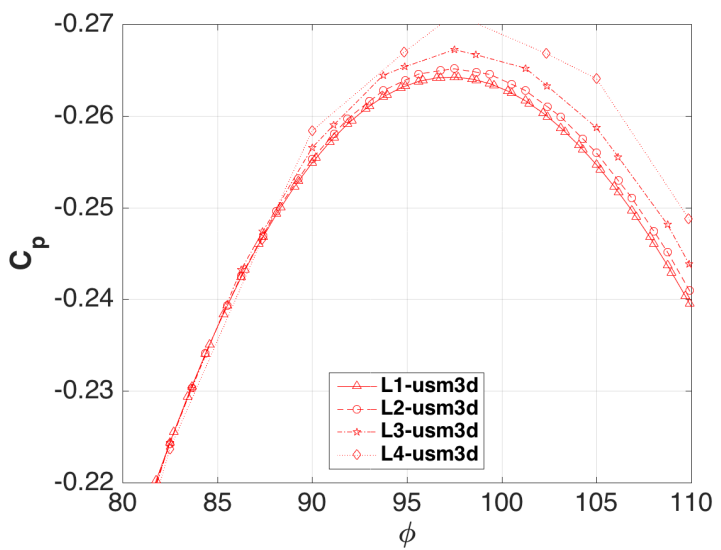

(c) USM3D

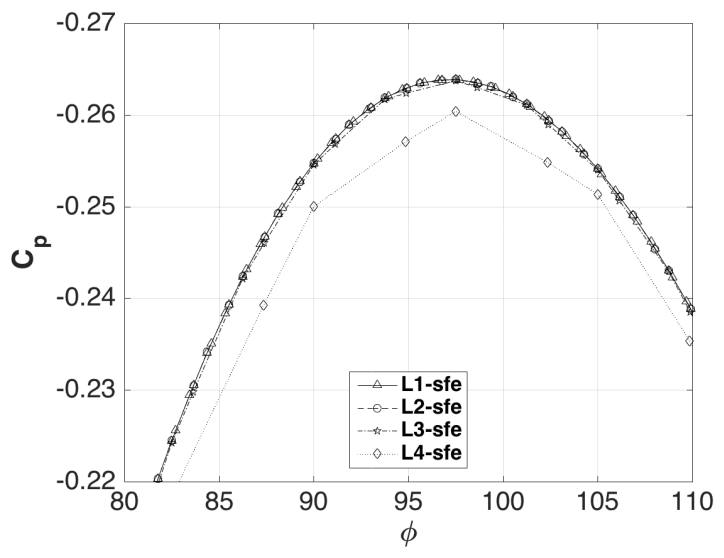

(b) SFE

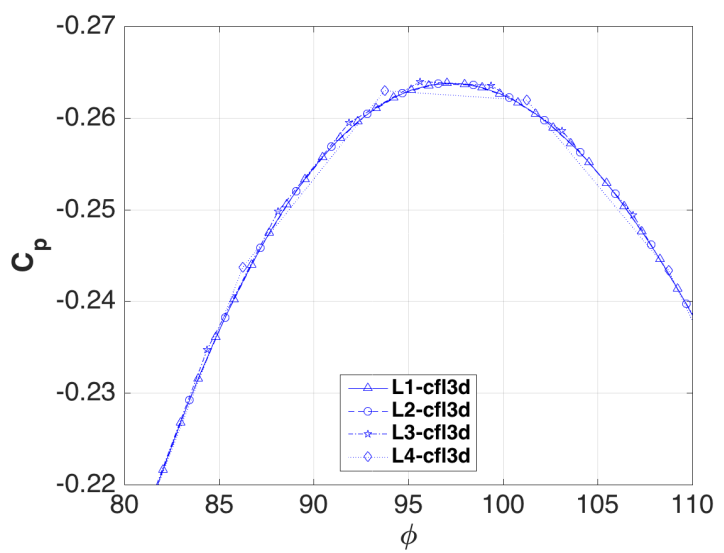

(d) CFL3D

Figure 17: Hemisphere cylinder zoomed view: grid convergence of surface pressure on $x=5$ plane near minimum location. 


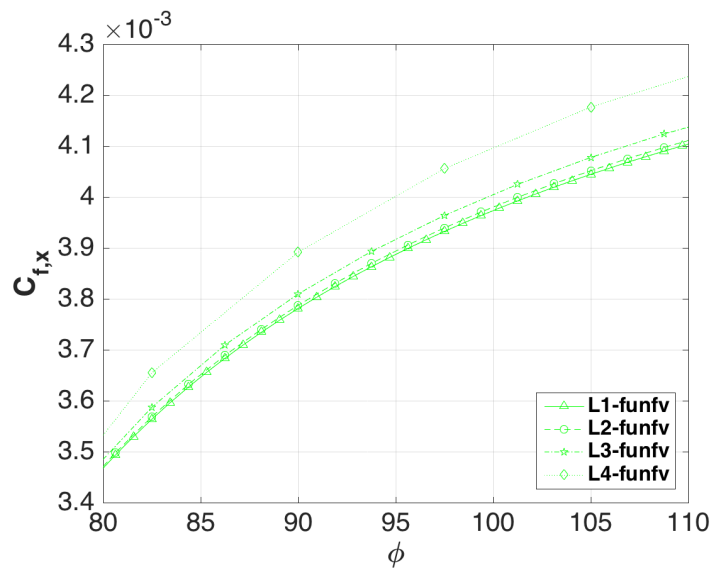

(a) FUNFV

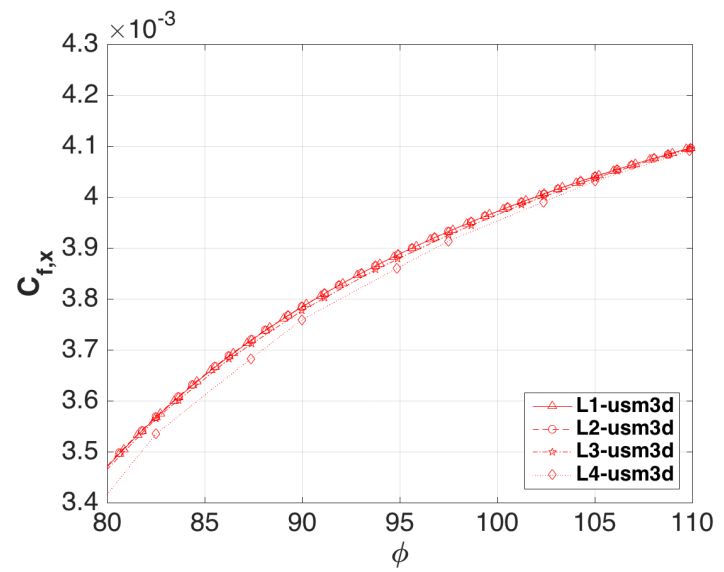

(c) USM3D

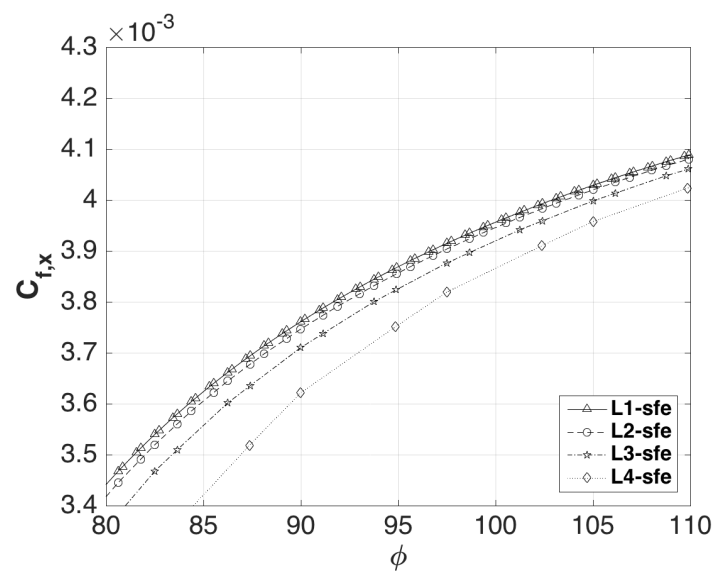

(b) SFE

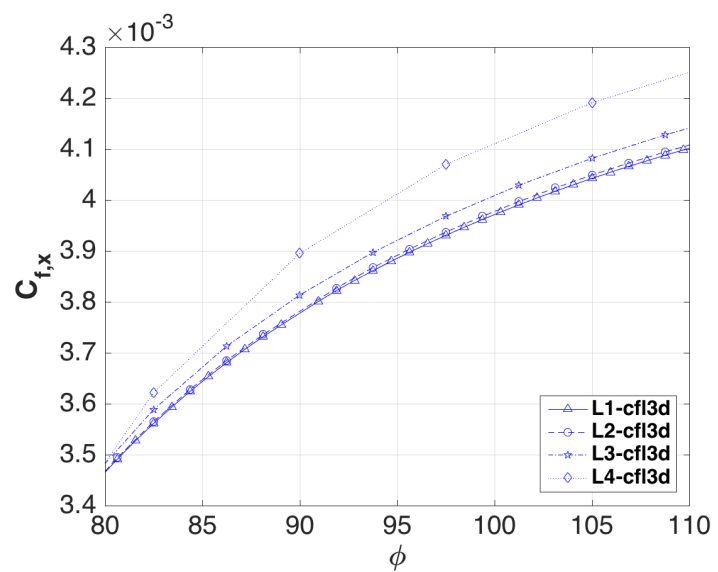

(d) CFL3D

Figure 18: Hemisphere cylinder zoomed view: grid convergence of $x$-component of skin friction on $x=5$ plane near minimum-pressure location.

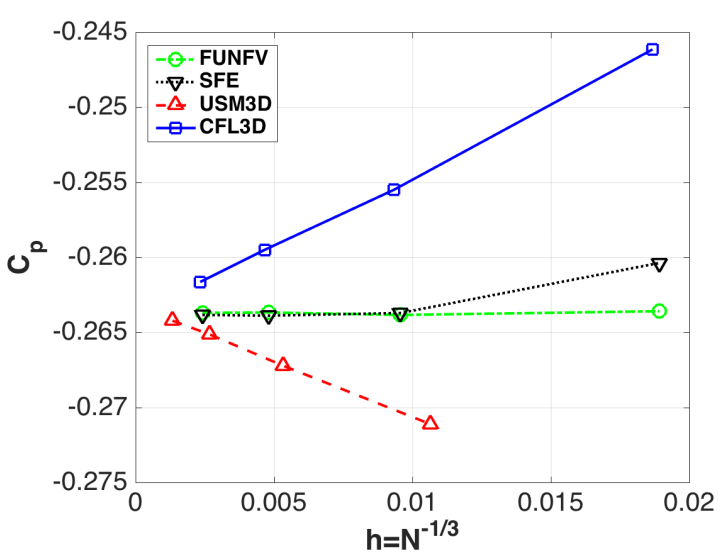

(a) Pressure

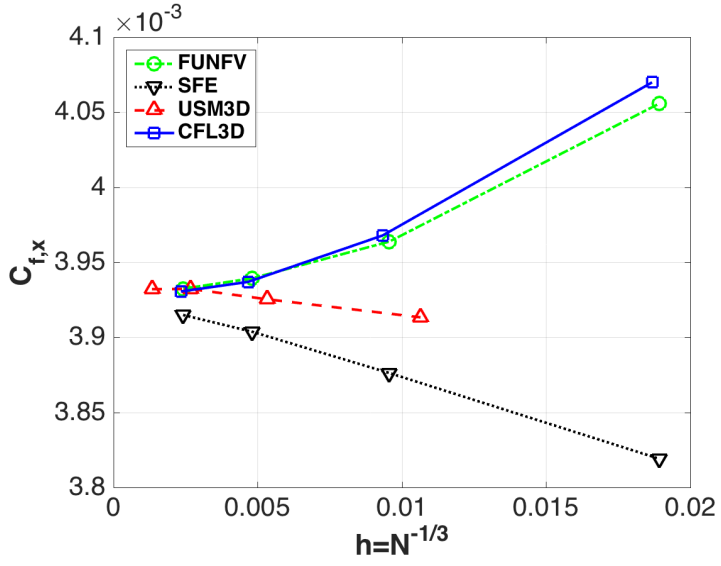

(b) Skin friction, $x$-component

Figure 19: Hemisphere cylinder: grid convergence of surface pressure and $x$-component of skin friction on $x=5$ plane near minimum pressure location, at $\phi=97.5^{\circ}$. 


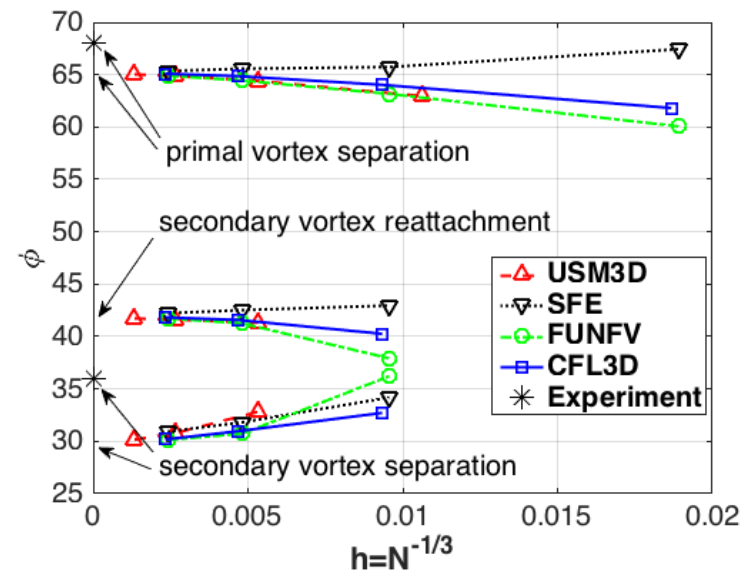

Figure 20: Grid convergence of vortex separation and reattachment locations for primal and secondary vortices at $x=5$ plane.

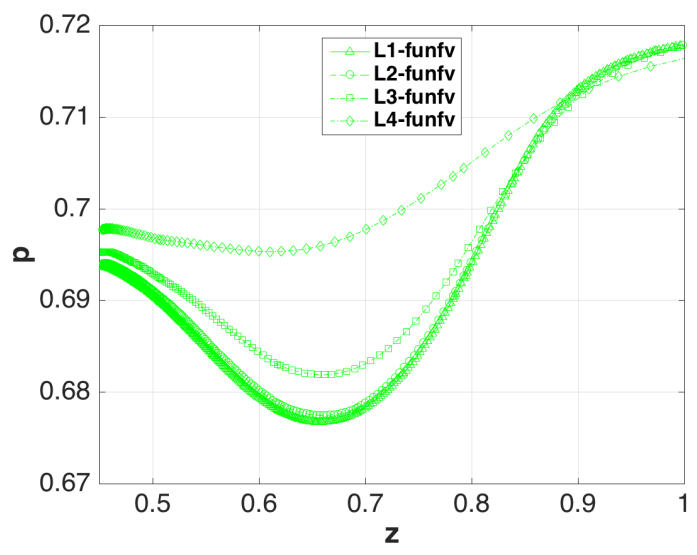

(a) FUNFV

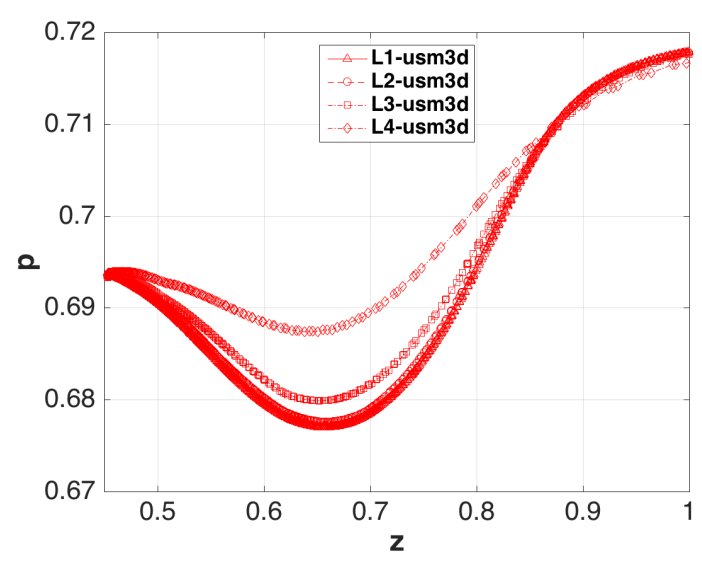

(c) USM3D

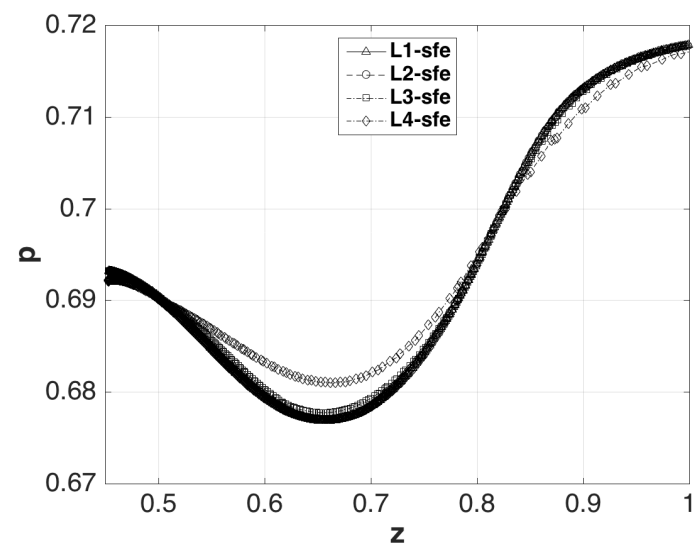

(b) SFE

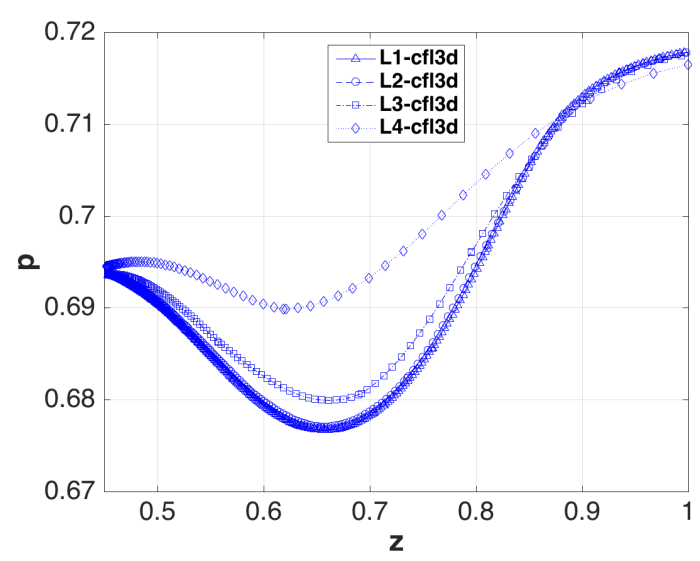

(d) CFL3D

Figure 21: Hemisphere cylinder zoomed view: grid convergence of off-body pressure near the upper surface along vertical line corresponding to $x=5.0$ and $y=0.21$. 


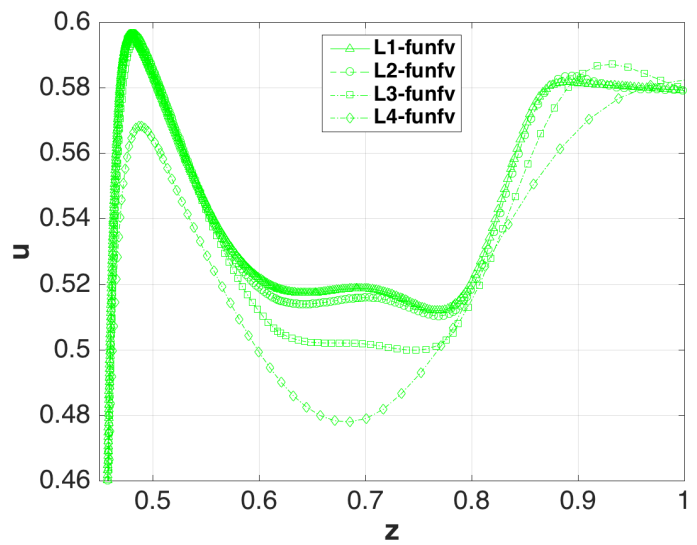

(a) FUNFV

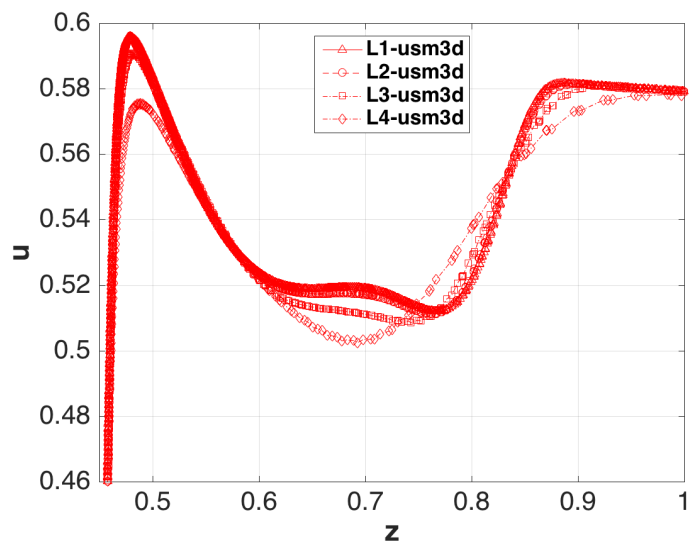

(c) USM3D

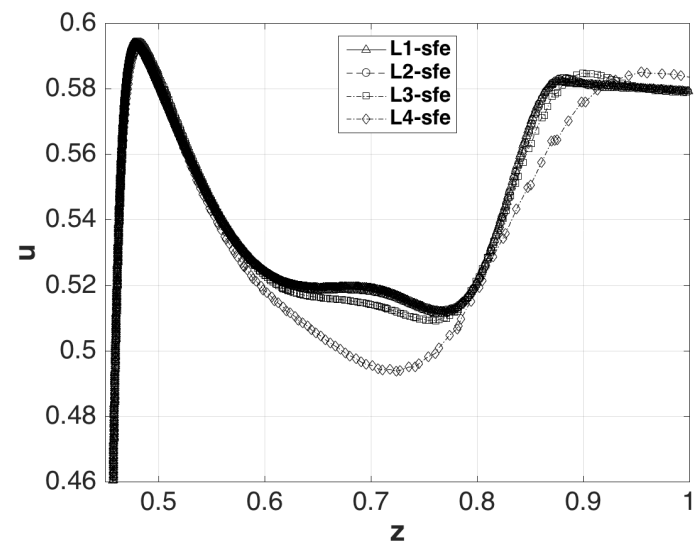

(b) SFE

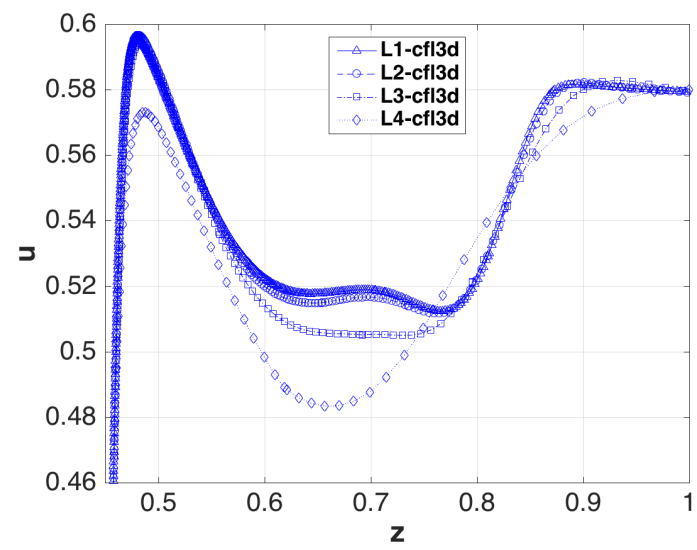

(d) CFL3D

Figure 22: Hemisphere cylinder zoomed view: grid convergence of off-body $u$-velocity near the upper surface along vertical line corresponding to $x=5.0$ and $y=0.21$. 


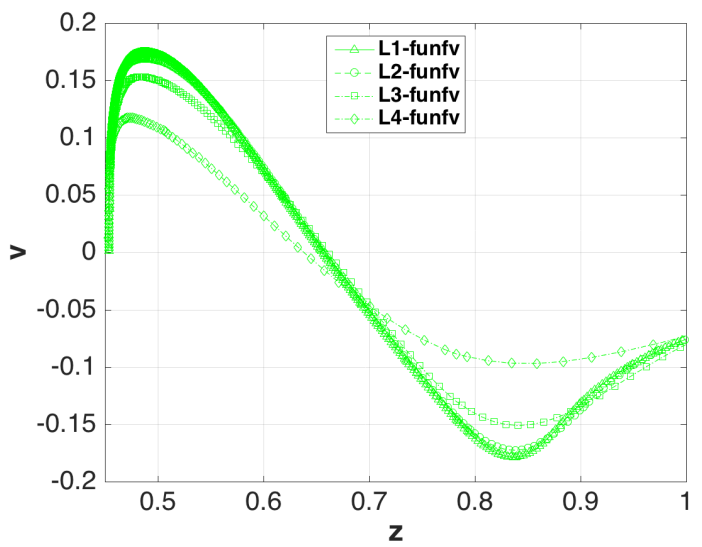

(a) FUNFV

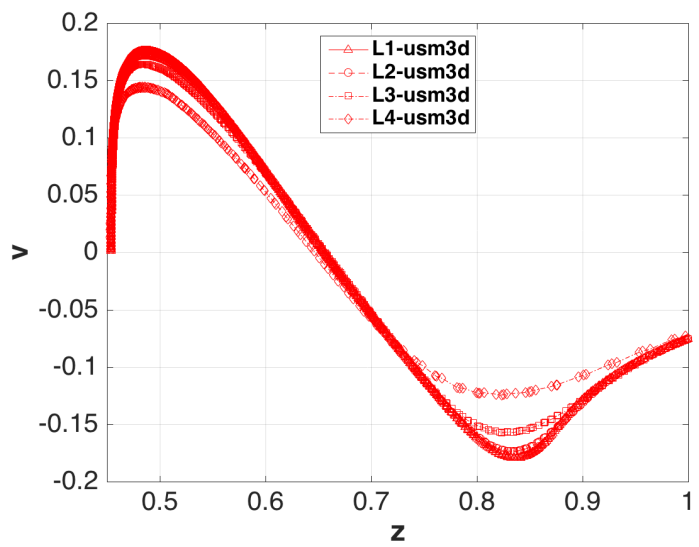

(c) USM3D

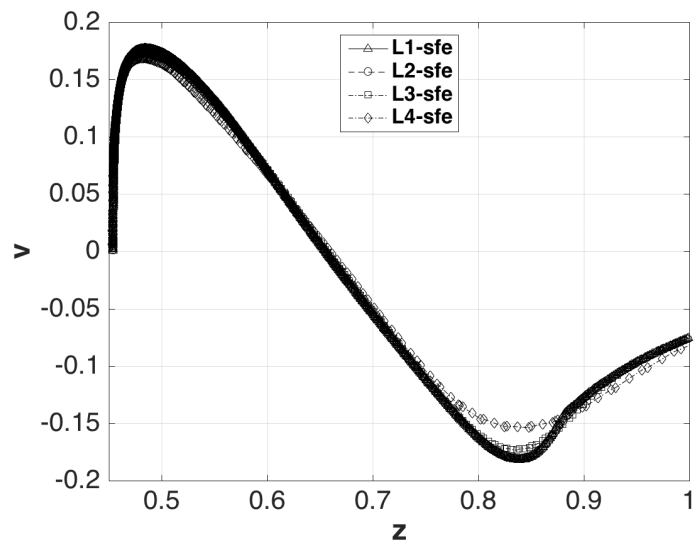

(b) SFE

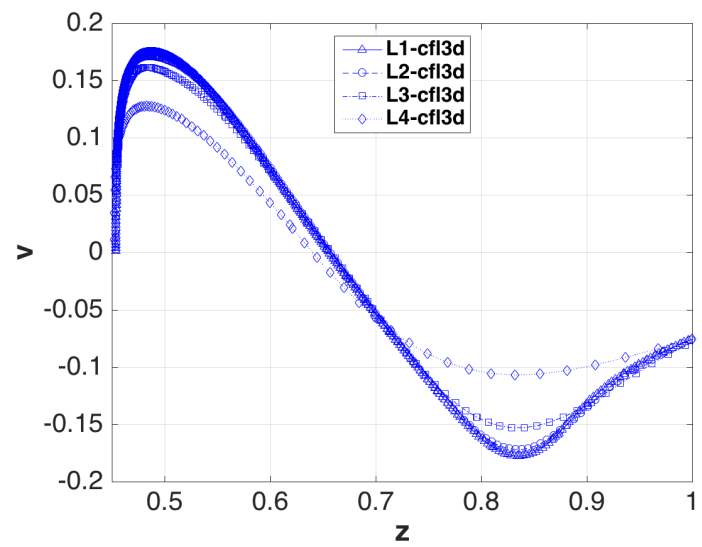

(d) CFL3D

Figure 23: Hemisphere cylinder zoomed view: grid convergence of off-body $v$-velocity near the upper surface along vertical line corresponding to $x=5.0$ and $y=0.21$. 


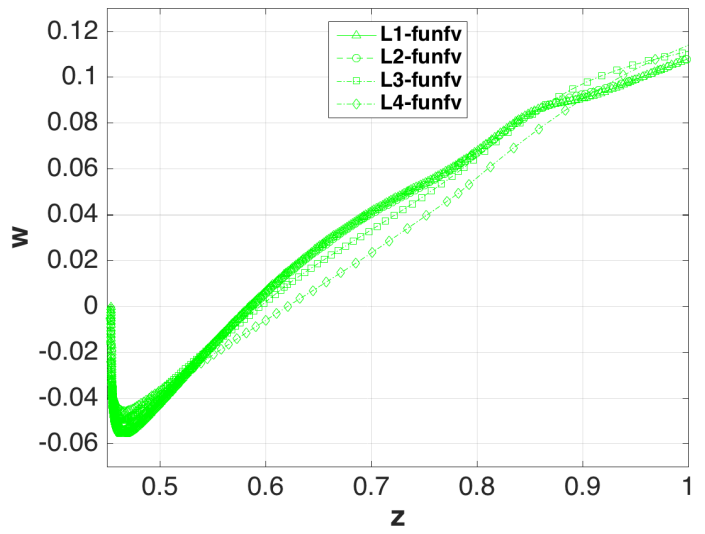

(a) FUNFV

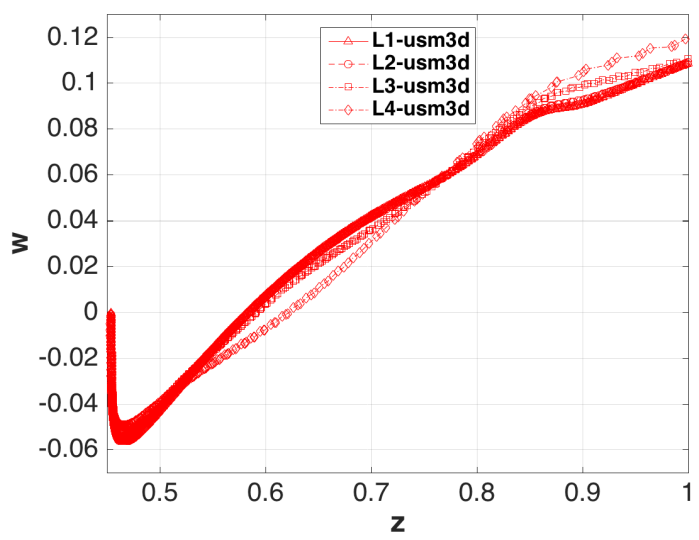

(c) USM3D

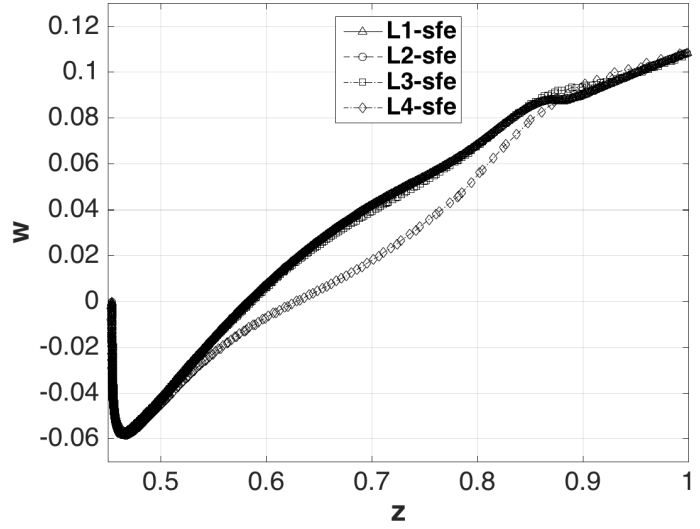

(b) SFE

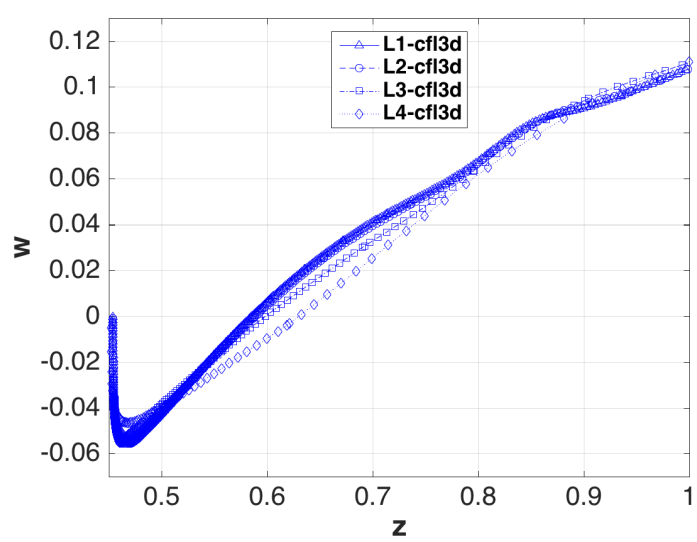

(d) CFL3D

Figure 24: Hemisphere cylinder zoomed view: grid convergence of off-body $w$-velocity near the upper surface along vertical line corresponding to $x=5.0$ and $y=0.21$. 


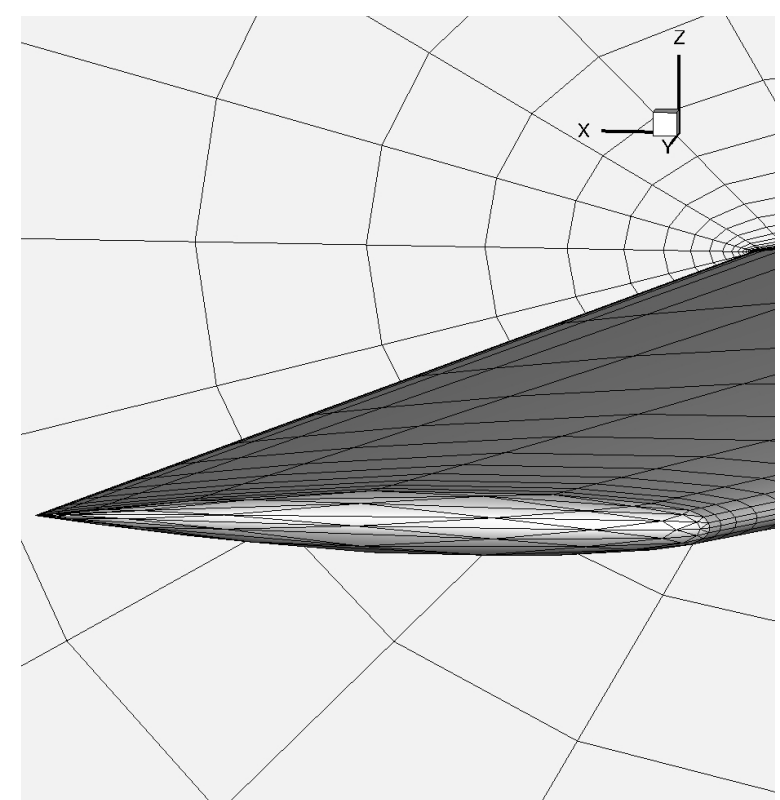

(a) Grid family 4 (prism/hex)

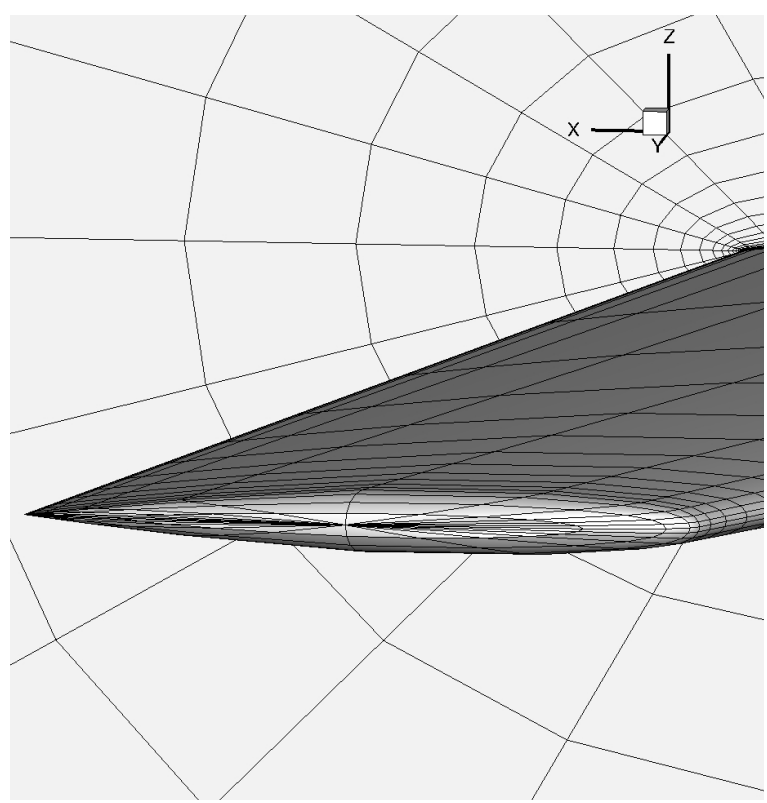

(b) Grid family 5 (structured)

Figure 25: OM6 wing tip surface. 


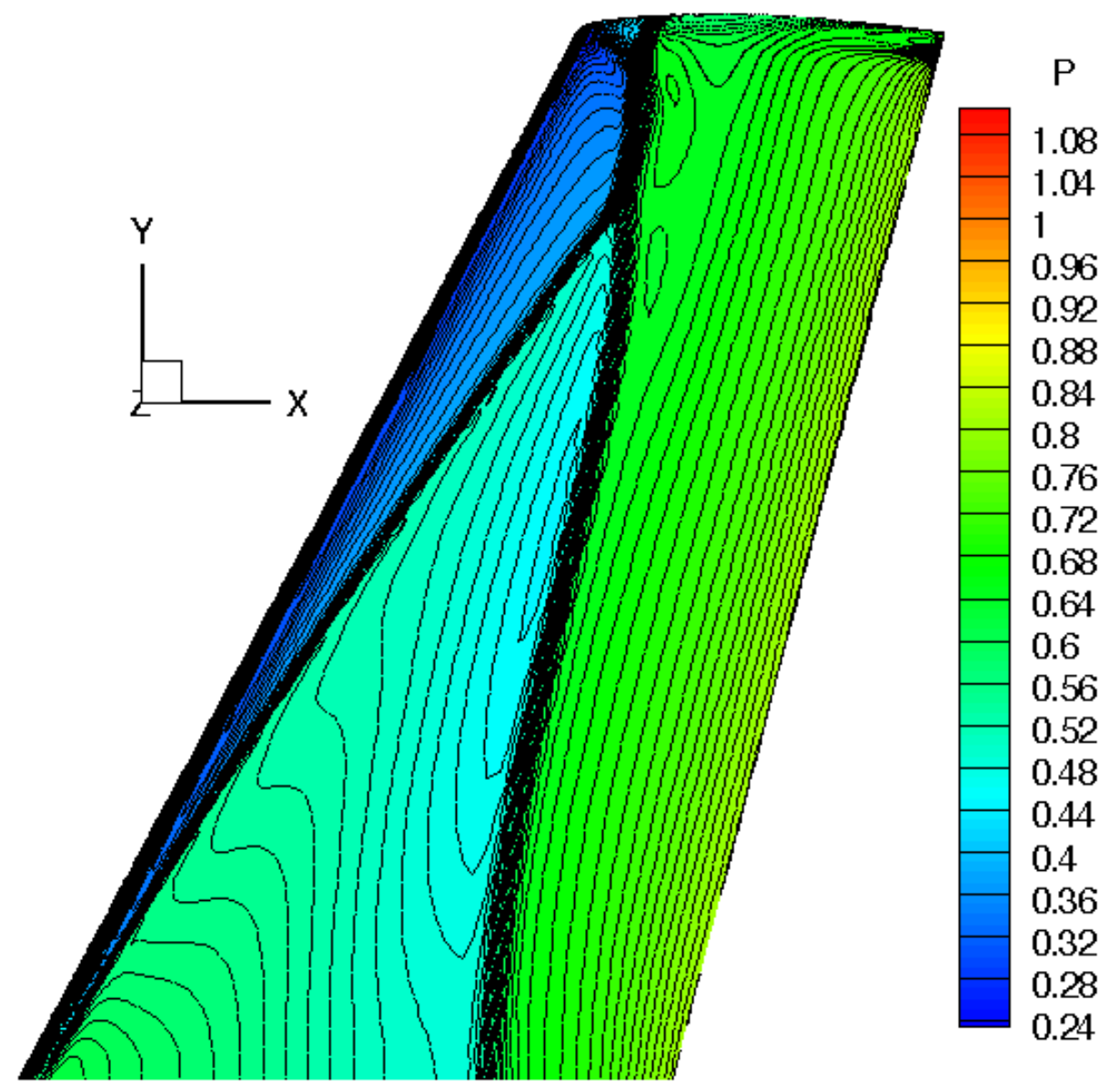

Figure 26: OM6 wing: pressure contours computed by USM3D on family 4 prism/hex L1 grid. 


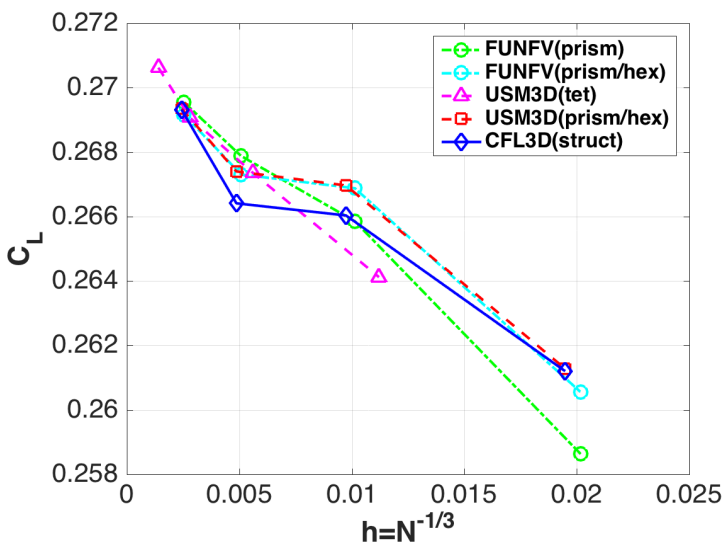

(a) Lift

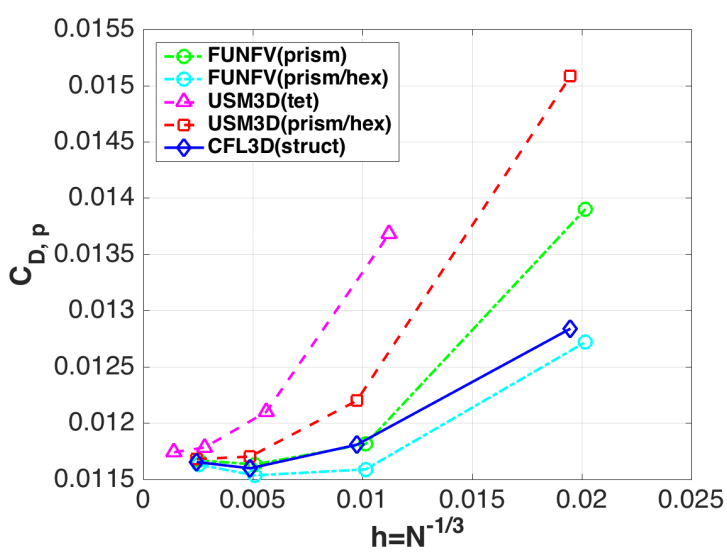

(c) Pressure drag

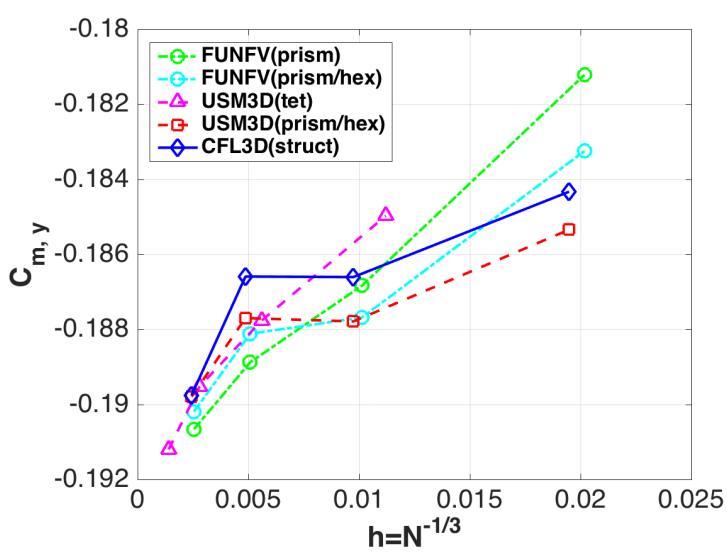

(e) Pitching moment

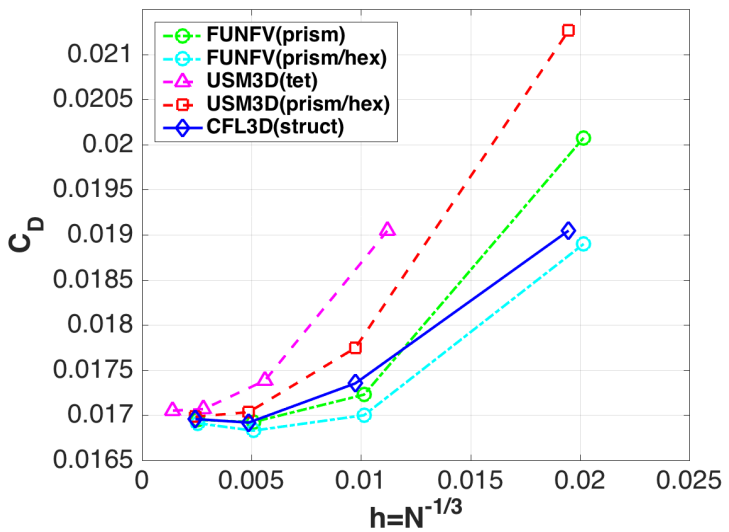

(b) Total drag

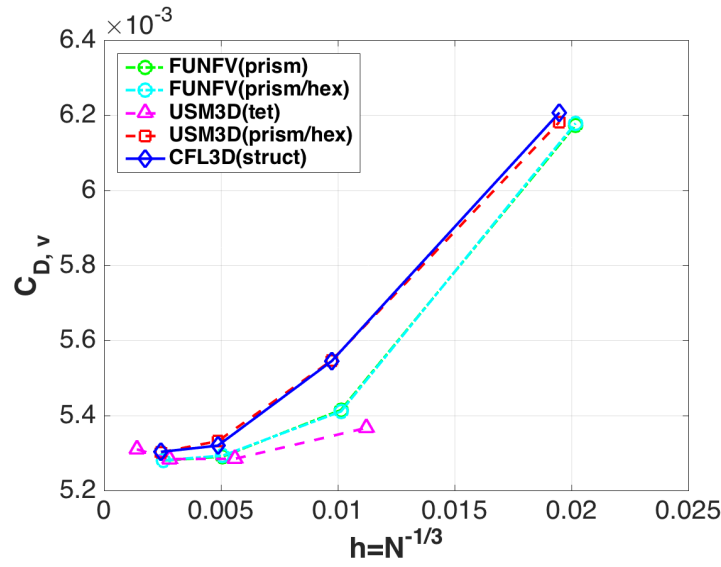

(d) Viscous drag

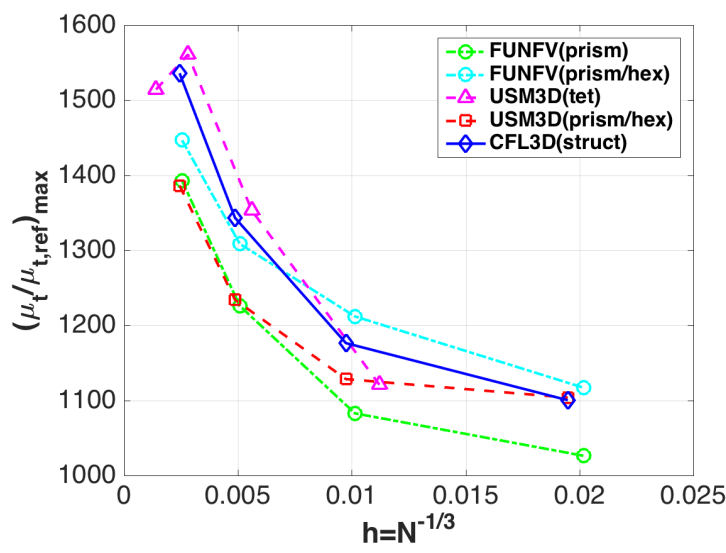

(f) Maximum eddy viscosity

Figure 27: OM6 wing: grid convergence of aerodynamic forces, pitching moment, and maximum eddy viscosity. 


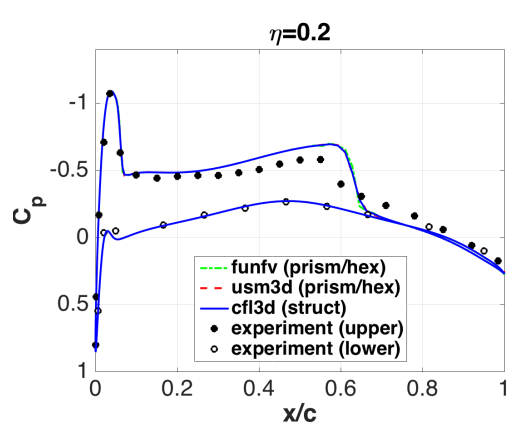

(a) Section 1

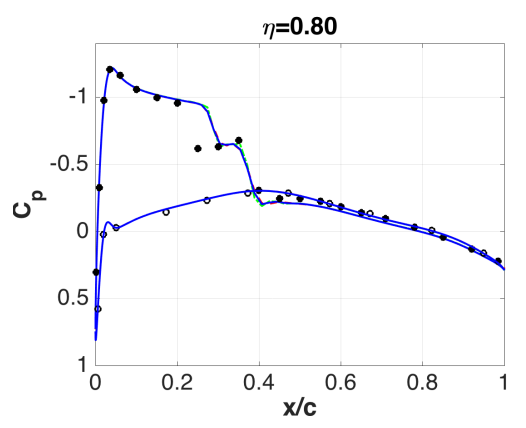

(d) Section 4

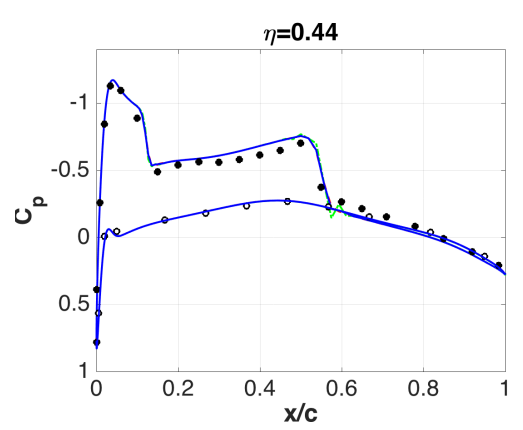

(b) Section 2

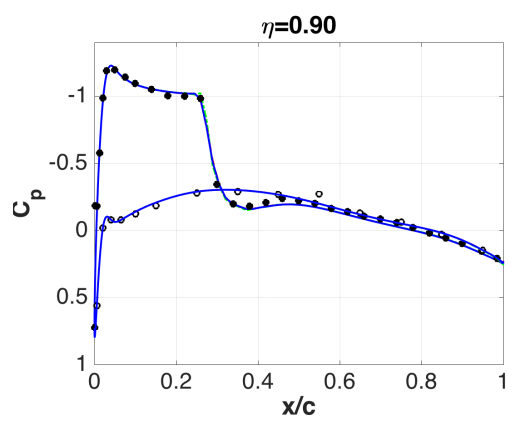

(e) Section 5

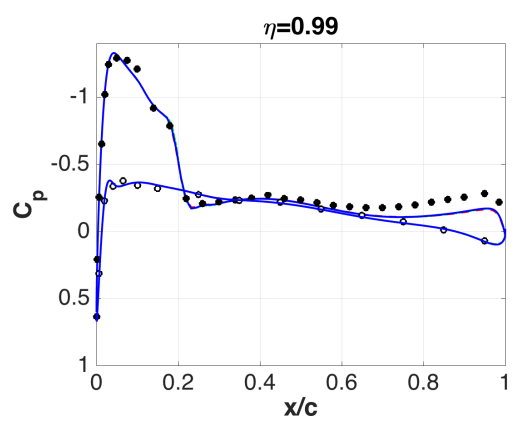

(g) Section 7

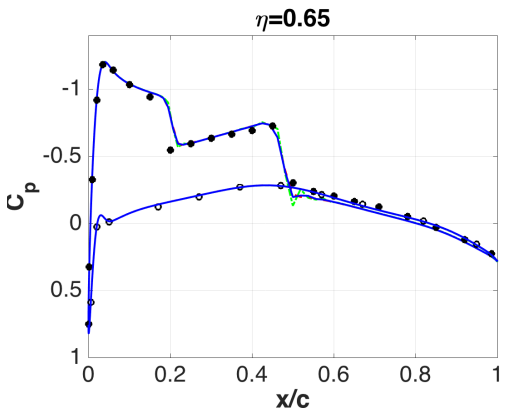

(c) Section 3

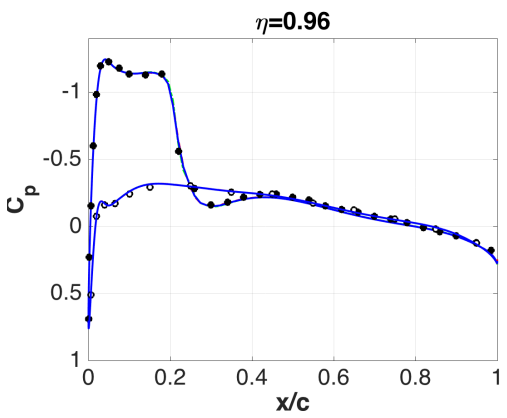

(f) Section 6

Figure 28: OM6 wing: variation of L1 grid surface pressure.

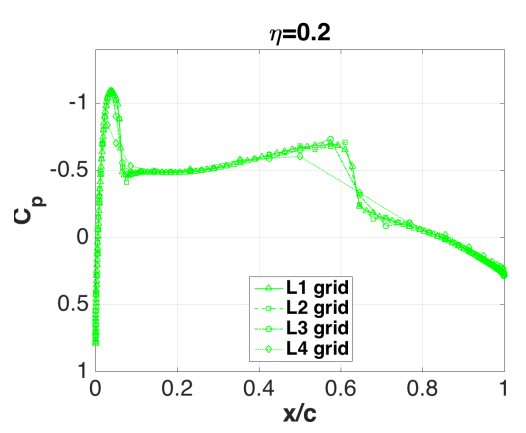

(a) FUNFV

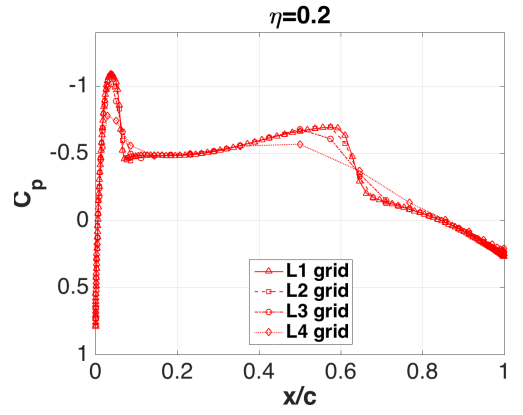

(b) USM3D

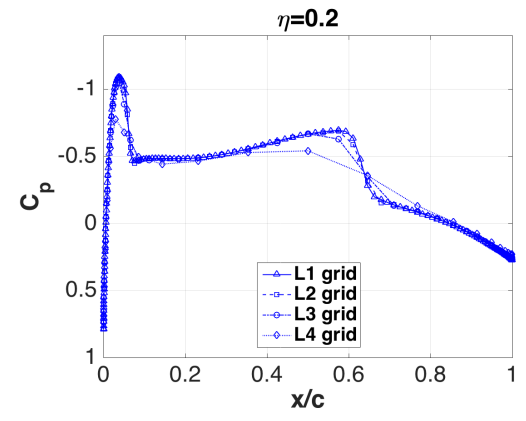

(c) CFL3D

Figure 29: OM6 section 1: global view of leeside pressure grid refinement. 


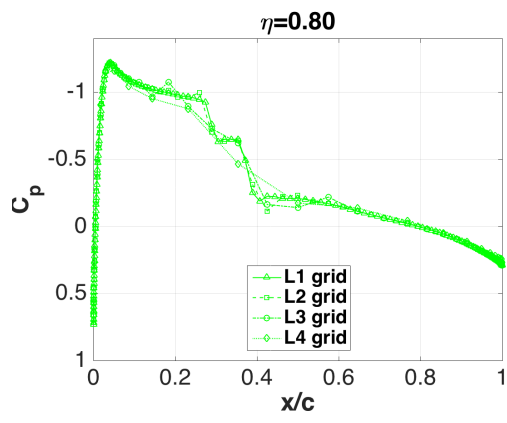

(a) FUNFV

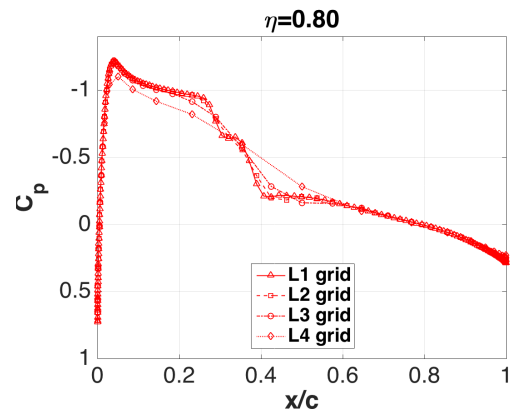

(b) USM3D

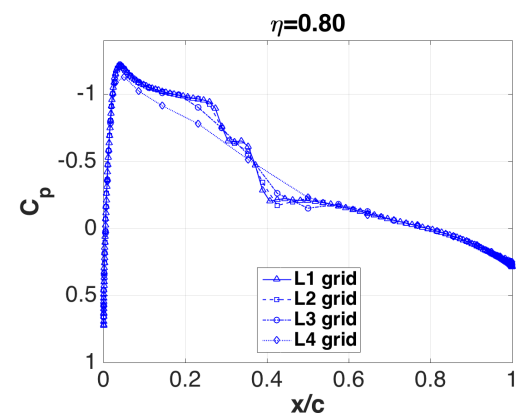

(c) CFL3D

Figure 30: OM6 section 4: global view of leeside pressure grid refinement.

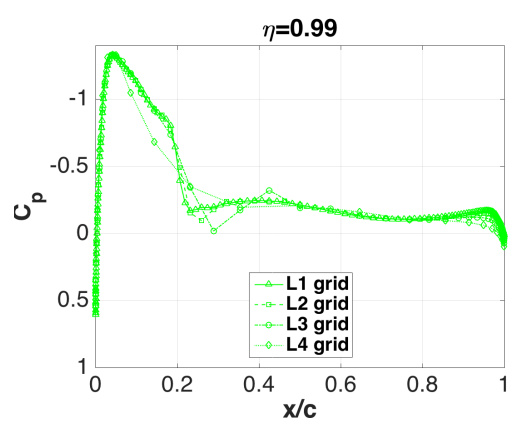

(a) FUNFV

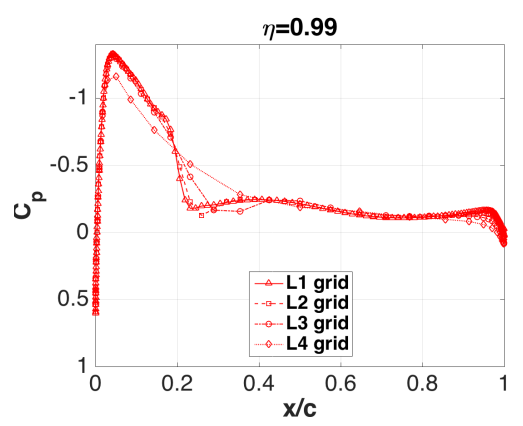

(b) USM3D

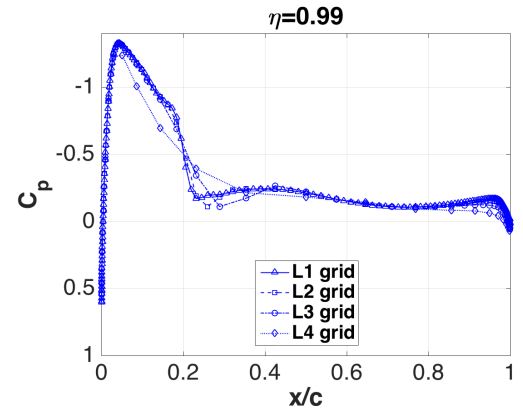

(c) CFL3D

Figure 31: OM6 section 7: global view of of leeside pressure grid refinement.

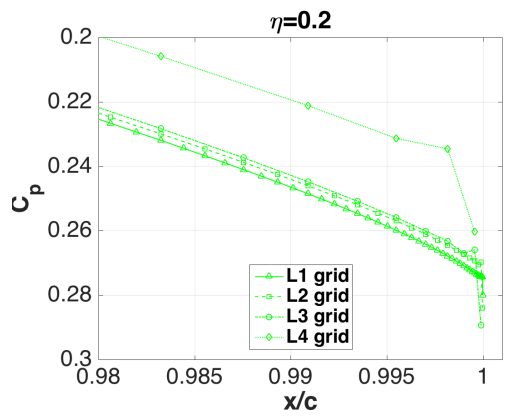

(a) FUNFV

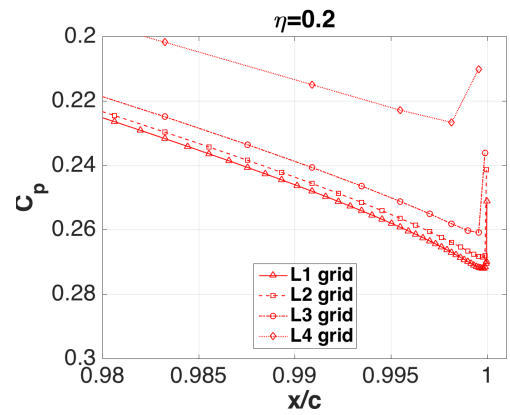

(b) USM3D

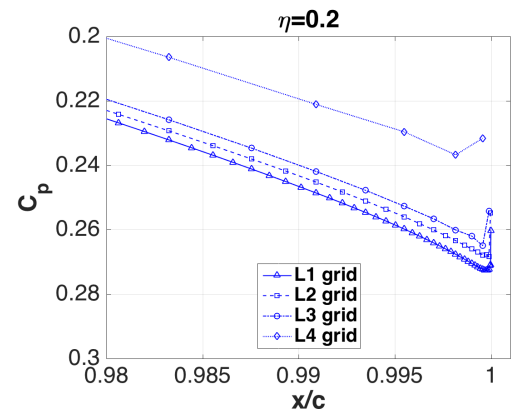

(c) CFL3D

Figure 32: OM6 section 1: zoom on leeside pressure grid refinement near trailing edge.

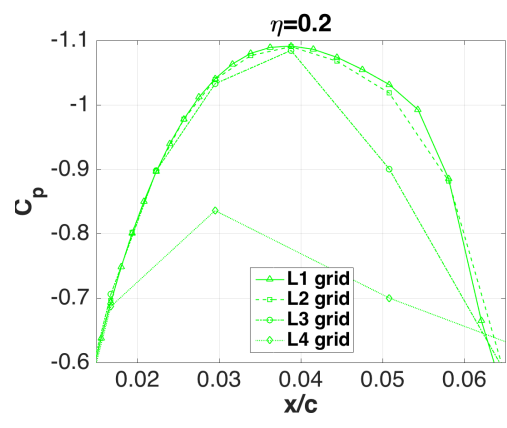

(a) FUNFV

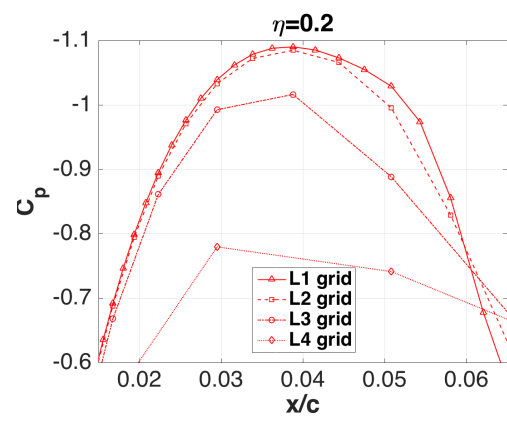

(b) USM3D

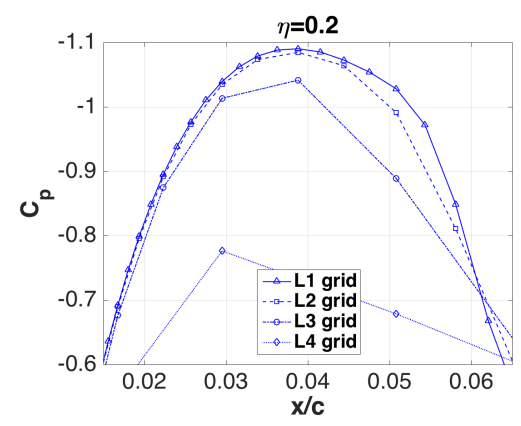

(c) CFL3D

Figure 33: OM6 section 1: zoom on grid refinement near minimum-pressure location. 


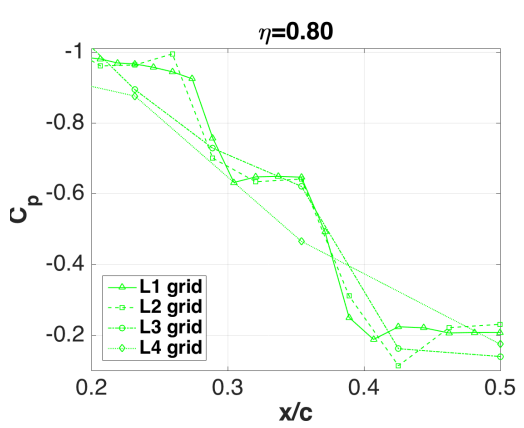

(a) FUNFV

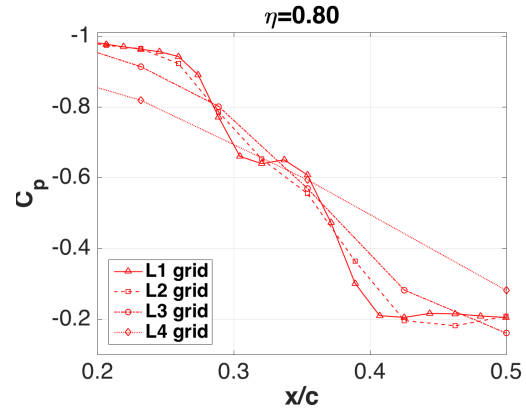

(b) USM3D

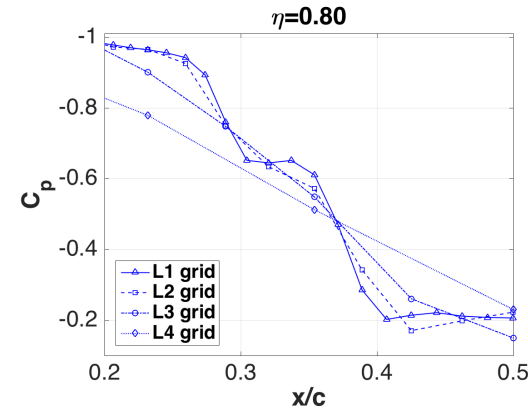

(c) CFL3D

Figure 34: OM6 section 4: zoom on grid refinement near shock intersection.

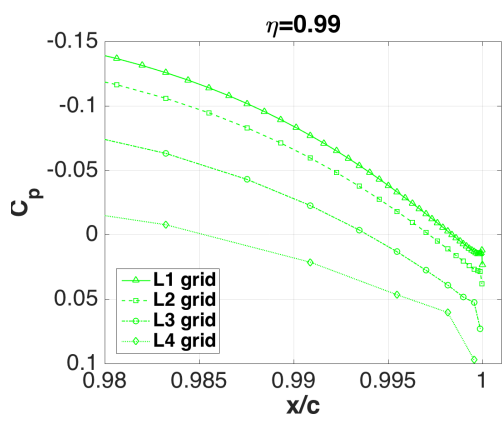

(a) FUNFV

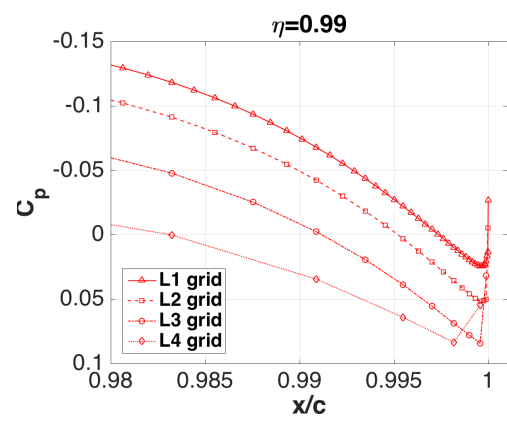

(b) USM3D

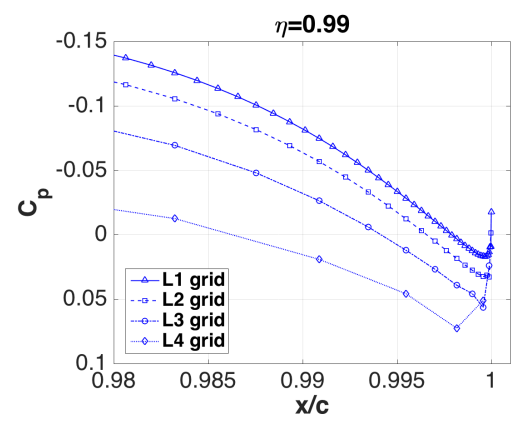

(c) CFL3D

Figure 35: OM6 section 7: zoom on leeside pressure grid refinement near trailing edge.

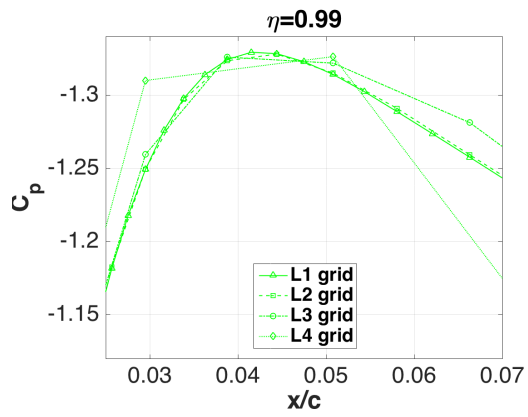

(a) FUNFV

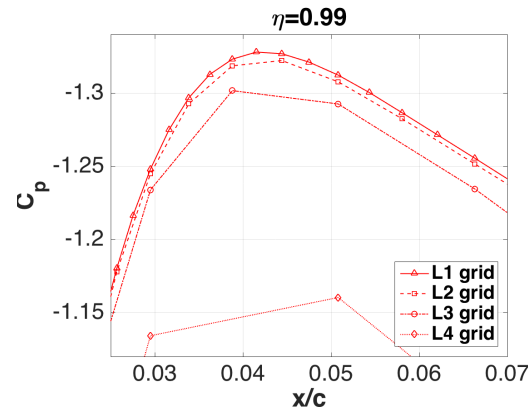

(b) USM3D

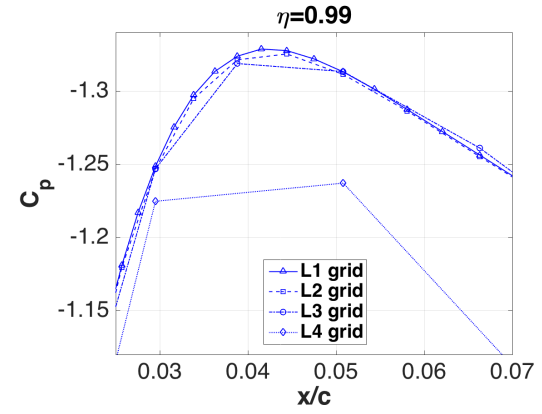

(c) CFL3D

Figure 36: OM6 section 7: zoom on grid refinement near minimum-pressure location. 